\title{
Charge and spin transport in strongly correlated one-dimensional quantum systems driven far from equilibrium
}

\author{
Giuliano Benenti, ${ }^{1,2}$ Giulio Casati, $, 2,3$ Tomaž Prosen, ${ }^{4}$ Davide Rossini, ${ }^{5}$ and Marko Žnidarič ${ }^{4}$ \\ ${ }^{1}$ Center for Nonlinear and Complex Systems, CNISM, and CNR-INFM, Università degli Studi dell'Insubria, Via Valleggio 11, \\ 22100 Como, Italy \\ ${ }^{2}$ Istituto Nazionale di Fisica Nucleare-Sezione di Milano, Via Celoria 16, 20133 Milano, Italy \\ ${ }^{3}$ Centre for Quantum Technologies, National University of Singapore, 117543 Singapore, Singapore \\ ${ }^{4}$ Department of Physics, Faculty of Mathematics and Physics, University of Ljubljana, SI-1000 Ljubljana, Slovenia \\ ${ }^{5}$ International School for Advanced Studies (SISSA), Via Beirut 2-4, I-34014 Trieste, Italy
}

(Received 14 January 2009; revised manuscript received 10 June 2009; published 8 July 2009)

\begin{abstract}
We study the charge conductivity in one-dimensional prototype models of interacting particles, such as the Hubbard and the $t-V$ spinless fermion models, when coupled to some external baths injecting and extracting particles at the boundaries. We show that, if these systems are driven far from equilibrium, a negative differential conductivity regime can arise. The above electronic models can be mapped into Heisenberg-like spin ladders coupled to two magnetic baths, so that charge transport mechanisms are explained in terms of quantum spin transport. The negative differential conductivity is due to oppositely polarized ferromagnetic domains that arise at the edges of the chain and therefore inhibit spin transport: we propose a qualitative understanding of the phenomenon by analyzing the localization of one-magnon excitations created at the borders of a ferromagnetic region. We also show that negative differential conductivity is stable against breaking of integrability. Numerical simulations of nonequilibrium time evolution have been performed by employing a Monte Carlo wave function approach and a matrix product operator formalism.
\end{abstract}

DOI: $10.1103 /$ PhysRevB.80.035110

PACS number(s): 75.10.Pq, 05.30.-d, 05.60. $-\mathrm{k}, 03.65 . \mathrm{Yz}$

\section{INTRODUCTION}

Transport properties of strongly interacting fermions in microscopic models of one-dimensional quantum systems have been the subject of a large number of theoretical and experimental studies. ${ }^{1}$ In the last few years this has become a topical subject, due to the rapidly developing process of miniaturization in semiconductor microelectronic devices that is approaching its natural limits, reaching the atomic or molecular scale. ${ }^{2-4}$ Of course, a technological breakthrough in this direction would require conceptually new devices, such as few or even single molecules embedded between electrodes, which could perform the basic functions of microelectronics. The first promising step in the realization of such devices comes from the observation of many-body effects, such as the Coulomb blockade and the Kondo effect in nanometer-scale systems, such as single molecules or carbon nanotubes. ${ }^{5,6}$ Establishing and reaching a suitable degree of control of nonlinear electronic transport, such as a negative differential conductivity (NDC) regime, would be one of the ultimate tasks for functional nanodevices since it lays at the basis of current rectification and amplification. NDC has been observed in a variety of nanoscopic objects, such as semiconductor quantum dots ${ }^{7}$ and carbon nanotubes, ${ }^{8}$ as well as single molecules. ${ }^{9}$

From a theoretical point of view, nonlinear transport properties in such systems are usually studied by considering effective models of few single-particle levels (see, e.g., Refs. 10-14). In this paper we adopt a rather different perspective and study the full many-body quantum dynamics of onedimensional prototype models of strongly interacting fermions when they are coupled to some external baths. We will show how effects of nonlinear transport naturally emerge in far-from-equilibrium situations by exploiting the many-body dynamics of such microscopic models in its whole complexity. While situations close to equilibrium are quite well understood and can be tackled by the powerful linear response formalism, ${ }^{15-21}$ almost nothing is known about the physics of such systems far from equilibrium. In this regime new quantum phases and phenomena can appear, thus making the problem relevant also for fundamental physics. ${ }^{22}$ Furthermore, the study of far-from-equilibrium quantum systems is of interest also for issues such as the control of heat flow at the nanoscale ${ }^{23,24}$ and, in quantum information processing, for quantum state preparation or transfer. ${ }^{25}$ Unfortunately a fully analytical treatment is generally unfeasible, ${ }^{26}$ and one typically has to resort to numerical simulations, aimed at solving the quantum master equation ${ }^{27-33}$ or based on different approaches, such as path integral Monte Carlo approach, ${ }^{34}$ time-dependent density matrix renormalization group, or current density functional theory. ${ }^{35-37}$

In this paper we consider two prototype microscopic onedimensional models of interacting fermions, namely, the Hubbard model and the $t$ - $V$ spinless fermion model, and couple them to some external baths that inject and extract particles at the system edges, thus mimicking the effect of electrodes. The Hubbard and the $t-V$ models can be mapped into the Heisenberg spin-1/2 ladder and chain, respectively. In these spin models NDC reflects in the suppression of spin conduction, while the operators injecting and extracting electrons are mapped into operators flipping the two spin species at the border of the chain. As an example of spin chains coupled to such "magnetic baths" one can consider molecular spin wires 38,39 with each boundary coupled to an external spin (magnetic impurity); the ratio of up-down and down-up spin-flip probabilities is determined by the populations of 
such impurities, which in turn can be tuned by means of applied electromagnetic fields. In the linear response regime, the electronic (i.e., fermionic) transport and correspondingly the spin transport can be ballistic or diffusive, depending on the values of the Hamiltonian parameters. Here we focus on the far-from-equilibrium regime beyond the linear response regime. Our numerical results show that, strikingly, in the above mentioned models it is possible to achieve a regime where charge or spin conductivity exhibits a negative differential with respect to the driving strength. NDC arises as a result of the appearance of a far-from-equilibrium steady state characterized, for the spin chain models, by long-range spin ordering into ferromagnetic domains. These ferromagnetic domains correspond to charge separation in the fermionic models, with all the electrons frozen in half of the lattice. In both cases, it is clear that such cooperative manybody state hampers spin flips (or charge injection or extraction), thus strongly suppressing the current. We will show that our numerical results can be qualitatively explained in terms of localization of one-magnon excitations.

The paper is organized as follows: in Sec. II we start by setting our electron transport problem and reducing it to a Lindblad master equation formalism, which will be used throughout the paper. In Sec. III we introduce the model of open Hubbard chain coupled to two macroscopic reservoirs and discuss some peculiar charge transport properties, focusing on the NDC behavior. In Sec. IV we consider a simplified model for spinless fermions and show that it can be mapped into a Heisenberg spin chain. In Sec. V we study in detail the spin transport properties of the Heisenberg chain. Moreover, we provide a one-magnon-localization argument that qualitatively explains the observed NDC behavior. To explore the possibility that our system undergoes a metalinsulator phase transition when driven far from equilibrium, we propose to study steady-state spin-spin correlation functions. We also add, in Sec. VI, a staggered magnetic field and check that NDC is stable against breaking of integrability. Finally, in Sec. VII we draw our conclusions. In Appendixes $\mathrm{A}$ and $\mathrm{D}$ we describe the two numerical methods used throughout the paper, namely, the quantum trajectory (QT) approach and the matrix product operator (MPO) formalism (Appendix A), give technical details on the mapping of our fermionic systems into spin chain models (Appendix B), provide some numerical results about the steady-state spin-spin correlation functions (Appendix C), and present an analytical derivation of the one-magnon argument for the Heisenberg spin chain (Appendix D). A brief account of the NDC features of the Heisenberg chain can be found in a recent paper by some of us. ${ }^{32}$

\section{MASTER EQUATION APPROACH}

Our electronic transport model is described by the Hamiltonian

$$
\mathcal{H}=\mathcal{H}_{S}+\mathcal{H}_{l}+\mathcal{H}_{c},
$$

where the different terms correspond to the nanoscale electronic system, the leads, and the lead-system coupling, respectively.

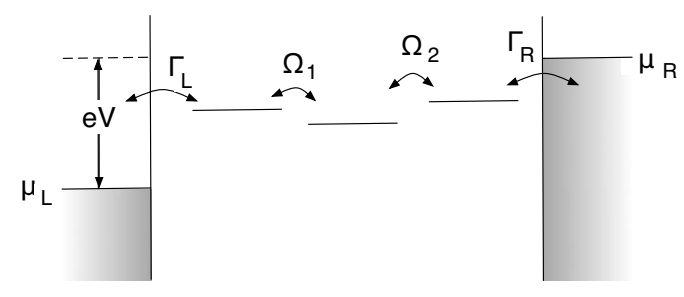

FIG. 1. Schematic drawing of the level structure for a chain with $N=3$ sites. We assume that the lead-chain tunneling rates $\Gamma_{L}, \Gamma_{R}$ are much larger than the intrachain tunneling rates $\Omega_{1}, \Omega_{2}$. The electronic current flows from the right lead (emitter) to the left lead (collector).

As sketched in Fig. 1, we consider a $N$-site chain, whose autonomous dynamics is described by Hamiltonian $\mathcal{H}_{S}$. Such a lattice models a nanoscale system, for instance, a chain of coupled quantum dots or a molecular wire (in the latter case, each lattice site corresponds to one atom).

The first and the last sites of the chain, 1 and $N$, are coupled to the left and right leads via the tunneling Hamiltonian

$$
\mathcal{H}_{c}=\sum_{k, s}\left(T_{L k} c_{L k, s}^{\dagger} c_{1, s}+T_{R k} c_{R k, s}^{\dagger} c_{N, s}\right)+\text { H.c. },
$$

where $c^{\dagger}, c$ are fermionic creation and annihilation operators: $c_{j s}^{\dagger}$ creates an electron with spin $s$ at site $j(j=1, \ldots, N$, $s=\uparrow, \downarrow)$ and $c_{L k, s}^{\dagger}\left(c_{R k, s}^{\dagger}\right)$ creates an electron in the left (right) lead in the state $|L k, s\rangle(|R k, s\rangle)$.

The leads are modeled as ideal Fermi gases,

$$
\mathcal{H}_{l}=\sum_{k, s} \epsilon_{k}\left(c_{L k, s}^{\dagger} c_{L k, s}+c_{R k, s}^{\dagger} c_{R k, s}\right),
$$

which are initially at equilibrium, at temperature $\mathcal{T}$ and chemical potentials $\mu_{L}, \mu_{R}=\mu_{L}+e V$, where $V$ is the applied bias voltage and $e$ is the electron charge. We assume that the coupling between the system and the leads is weak, such that the state $\rho_{B}(t)$ of the leads at any time $t$ is well described by $\rho_{B}(t)=\rho_{L} \otimes \rho_{R}$, with $\rho_{L}$ and $\rho_{R}$ grand canonical density matrices for the left and right leads, respectively.

A Lindblad master equation for the system's evolution can be obtained from our microscopic Hamiltonian model following standard textbook derivations under the usual BornMarkov and rotating wave approximations and neglecting the Lamb-type renormalization of the unperturbed energy levels (see, e.g., Sec. IIIC in Ref. 40),

$$
\frac{\partial \rho}{\partial t}=-\frac{i}{\hbar}\left[\mathcal{H}_{S}, \rho\right]-\frac{1}{2} \sum_{m}\left\{L_{m}^{\dagger} L_{m}, \rho\right\}+\sum_{m} L_{m} \rho L_{m}^{\dagger},
$$

where $\rho(t)$ is the density matrix describing the open quantum system, the Lindblad operators $L_{m}$ describe the effect of the environment, while $[\cdot, \cdot]$ and $\{\cdot, \cdot\}$ denote the commutator and the anticommutator, respectively. Hereafter we shall set $\hbar=1$. Moreover, we assume that the tunneling between site 1 $(N)$ and the left (right) lead is much faster than the intrachain tunneling and that we can neglect the effects of Coulomb repulsion on the system-lead transition rates. This is the case in the so-called wide-band limit, in which the conduction 
bandwidth of the leads is much larger than all other relevant energy scales and all the relevant lead states are located in the center of the conduction band, so that the energy dependence in the system-lead transition rate may be neglected. Under these approximations, we can easily derive the Lindblad master equation [Eq. (4)], with four Lindblad operators on each of the two chain ends,

$$
\begin{aligned}
& L_{1}=\sqrt{\Gamma_{L} f_{L}} c_{1, \uparrow}^{\dagger}, \quad L_{2}=\sqrt{\Gamma_{L}\left(1-f_{L}\right)} c_{1, \uparrow}, \\
& L_{3}=\sqrt{\Gamma_{L} f_{L}} c_{1, \downarrow}^{\dagger}, \quad L_{4}=\sqrt{\Gamma_{L}\left(1-f_{L}\right)} c_{1, \downarrow},
\end{aligned}
$$

and similarly

$$
\begin{aligned}
& L_{5}=\sqrt{\Gamma_{R} f_{R}} c_{N, \uparrow}^{\dagger}, \quad L_{6}=\sqrt{\Gamma_{R}\left(1-f_{R}\right)} c_{N, \uparrow}, \\
& L_{7}=\sqrt{\Gamma_{R} f_{R}} c_{N, \downarrow}^{\dagger}, \quad L_{8}=\sqrt{\Gamma_{R}\left(1-f_{R}\right)} c_{N, \downarrow},
\end{aligned}
$$

where

$$
\begin{array}{cc}
\Gamma_{L} \equiv 2 \pi\left|T_{L}\left(E_{1}\right)\right|^{2} g_{L}\left(E_{1}\right), & f_{L} \equiv f_{L}\left(E_{1}\right), \\
\Gamma_{R} \equiv 2 \pi\left|T_{R}\left(E_{N}\right)\right|^{2} g_{R}\left(E_{N}\right), & f_{R} \equiv f_{R}\left(E_{N}\right),
\end{array}
$$

with $E_{1}\left(E_{N}\right)$ being the energy difference between the two chain states involved in the transitions for site $1(N)$, $g_{l}(l=L, R)$ being the density of states of lead $l$ (we assume that the leads are macroscopic objects, with a continuous density of states), $T_{l}\left(\epsilon=\epsilon_{k}\right) \equiv T_{l k}$, and $f_{l}(\epsilon)=\left[1+e^{\left(\epsilon-\mu_{l}\right) / k_{B} \mathcal{T}}\right]^{-1}$ denoting the Fermi function, with $k_{B}$ as the Boltzmann constant. Note that the energy differences $E_{1}, E_{N}$ contain the charging energy $E_{c}$ if an electron is tunneling onto an already occupied site but does not contain it if the site is initially empty. We have neglected the dependence of the Fermi functions $f_{L}, f_{R}$ on $E_{c}$. This condition is fulfilled when $E_{c} \ll k_{B} \mathcal{T}$ (in the Hubbard and $t$ - $V$ models described in this paper such constraint corresponds to on-site repulsion $U \ll k_{B} \mathcal{T}$ and nearest-neighbor repulsion $V \ll k_{B} \mathcal{T}$, respectively). Finally, in order to consider incoherent tunneling of electrons into the chain (sequential tunneling approximation), the level broadening due to the chain-lead tunneling must be small compared to temperature, that is, we require $\Gamma_{L}, \Gamma_{R} \ll k_{B} \mathcal{T}$.

As we shall see in this paper, a main advantage of the master equation approach is that it can be applied far from equilibrium beyond linear response regime. The far-fromequilibrium regime in our model corresponds to large bias voltage, $e V \gg k_{B} \mathcal{T}$, with the energy differences $E_{1}, E_{N}$ such that $\mu_{L} \ll E_{1}, E_{N} \ll \mu_{R}$. In this limit, $f_{L} \rightarrow 0, f_{R} \rightarrow 1$, that is, the backward flow of electrons (against the applied bias) vanishes.

The master equation approach may be generalized, including the effects of Coulomb repulsion ${ }^{41}$ (thus describing the Coulomb blockade phenomenon) or the coupling of multilevel nanoscale systems to external leads. ${ }^{42,43}$ The price to pay for such generalizations is, in general, the introduction of a larger number of Lindblad operators, corresponding to all possible transitions between the levels of the nanosystem.

The main transport quantity, the electron current $j$, is defined by the continuity equation of the local charge density $n_{k, s} \equiv c_{k, s}^{\dagger} c_{k, s}, n_{k}=n_{k, \uparrow}+n_{k, \downarrow}$,

$$
\frac{\partial n_{k}}{\partial t}+\nabla j_{k}=0
$$

which can be rewritten as

$$
j_{k+1}-j_{k}=i\left[n_{k}, \mathcal{H}_{S}\right], \quad k=1, \ldots, N-1 .
$$

Note that, due to the continuity equation, one has $j=j_{k}$ for any $k$ along the chain.

By definition the electron current is given by

$$
\langle j\rangle=\frac{d N_{R}}{d t}=-\frac{d N_{L}}{d t},
$$

with $N_{R}\left(N_{L}\right)$ being the number of electrons in the right (left) lead. This equation expresses the current in terms of the number of electrons that enter the system from the left reservoir $\left(-d N_{L} / d t\right)$ or go out into the right reservoir $\left(d N_{R} / d t\right)$ per time unit. As we shall discuss in Appendix A, $-d N_{L} / d t$ and $d N_{R} / d t$ may be computed by means of the quantum trajectory approach. We will use both Eqs. (10) and (11) to compute the current.

Explicitly solvable models of master equations are very limited, therefore support from extensive numerical simulations is generally needed. We used two methods to face this problem. The first is a Monte Carlo wave function approach that is based on the technique of QTs, which is widely used in quantum optics. ${ }^{44-47}$ The second is a MPO technique based on the time-dependent density matrix renormalization group method. ${ }^{48-52}$ QTs revealed themselves a powerful tool in the study of relatively small system sizes, especially for situations with a strong external bias, where equilibration times needed to reach the steady state are generally long. On the other hand, the MPO method can deal with systems up to one order of magnitude larger, but it may encounter some difficulties in converging to the stationary state for large driving fields. Both numerical methods are briefly discussed in Appendix A.

\section{HUBBARD MODEL}

We start our analysis by considering a paradigmatic model for the physics of strongly interacting electronic systems: the Hubbard model. Its Hamiltonian is a sum of a kinetic term allowing for electron tunneling between the neighboring lattice sites and a potential term consisting of an on-site interaction; in one dimension it is given by

$$
\mathcal{H}_{S}=-t \sum_{j, s}\left(c_{j, s}^{\dagger} c_{j+1, s}+\text { H.c. }\right)+U \sum_{j=1}^{N} n_{j, \uparrow} n_{j, \downarrow},
$$

where $s$ stands for spin-up $\uparrow$ or spin-down $\downarrow$ configuration, while $j=1, \ldots, N$ is the site index and $N$ is the number of lattice sites. The operators $c_{j, s}^{\dagger}, c_{j, s}$ create and annihilate a spin-1/2 fermion with spin $s$ at site $j$ and satisfy the usual anticommutation rules; $n_{j, s}=c_{j, s}^{\dagger} c_{j, s}$ is the corresponding number operator. We consider open boundary conditions, therefore the sum over $j$ in the first term runs from 1 to $N-1$. The system parameters $t$ and $U(U>0)$ describe, respectively, the nearest-neighbor hopping strength and the on- 
site repulsion between electrons with opposite spins.

Both ends of the Hubbard chain are coupled to some electrodes that act on the system by injecting or extracting particles with different spins. In the Lindblad master equation formalism, we assume that their effect can be modeled by Lindblad operators (5) and (6).

From continuity Eq. (10) we obtain

$$
j=-t \sum_{s}\left(i c_{k, s}^{\dagger} c_{k+1, s}+\text { H.c. }\right) \quad(k=1, \ldots, N-1) .
$$

We examined the fermionic transport properties of Hubbard model (12) coupled to external baths by exploiting a mapping of this system into a spin ladder model, where the particle current is replaced by the spin current. Specifically, the Hamiltonian in Eq. (12) is mapped into a Heisenberg spin ladder by first employing a double Jordan-Wigner transformation (JWT) of spin-up and spin-down fermions (separately) into two different species of hard-core bosons. Then they are transformed into two species of spin-1/2 particles, which are described by the Pauli matrices $\sigma_{j}^{\alpha}$ and $\tau_{j}^{\alpha}$ $(\alpha=x, y, z)$. Details are given in Appendix B. One finally arrives at the following spin ladder Hamiltonian for the autonomous system:

$$
\begin{aligned}
\mathcal{H}_{S}= & -\frac{t}{2} \sum_{j=1}^{N-1}\left[\left(\sigma_{j}^{x} \sigma_{j+1}^{x}+\sigma_{j}^{y} \sigma_{j+1}^{y}\right)+\left(\tau_{j}^{x} \tau_{j+1}^{x}+\tau_{j}^{y} \tau_{j+1}^{y}\right)\right] \\
& +\frac{U}{4} \sum_{j=1}^{N}\left(\sigma_{j}^{z}+1\right)\left(\tau_{j}^{z}+1\right) .
\end{aligned}
$$

The Lindblad operators in Eqs. (5) and (6) correspond, in the spin-1/2 picture, to operators flipping the two spin species at the borders of the chain. Apart from some phase factor that is uninfluent for our purposes (see Appendix B) we have that $c_{j, \uparrow}^{\dagger} \rightarrow \sigma_{j}^{+}$and $c_{j, \uparrow} \rightarrow \sigma_{j}^{-}$for spin-up particles while $c_{j, \downarrow}^{\dagger} \rightarrow \tau_{j}^{+}$ and $c_{j, \downarrow} \rightarrow \tau_{j}^{-}$for spin-down particles $\left[\sigma_{j}^{ \pm} \equiv\left(\sigma_{j}^{x} \pm \mathrm{i} \sigma_{j}^{y}\right) / 2\right.$ and $\tau_{j}^{ \pm} \equiv\left(\tau_{j}^{x} \pm \mathrm{i} \tau_{j}^{y}\right) / 2$ denote the raising and lowering operators for the two spin species].

The spin current $j$ analogous to electron current (13) is derived from the continuity equation for the local spin operators $S_{k}^{z} \equiv \sigma_{k}^{z} / 2, \partial_{t} S_{k}^{z}+\nabla\left(j_{\sigma}\right)_{k}=0$, which can be rewritten as $\left(j_{\sigma}\right)_{k+1}-\left(j_{\sigma}\right)_{k}=\frac{i}{2}\left[\sigma_{k}^{z}, \mathcal{H}_{S}\right]$ [analogous equations can be written for the $\tau$ species in Eq. (14)]. We obtain

$$
\begin{gathered}
j \equiv j_{\sigma}+j_{\tau}, \\
j_{\sigma}=-\frac{t}{2}\left(\sigma_{k}^{x} \sigma_{k+1}^{y}-\sigma_{k}^{y} \sigma_{k+1}^{x}\right), \\
j_{\tau}=-\frac{t}{2}\left(\tau_{k}^{x} \tau_{k+1}^{y}-\tau_{k}^{y} \tau_{k+1}^{x}\right) .
\end{gathered}
$$

In the following, we choose a symmetric driving, $\Gamma_{L}=\Gamma_{R} \equiv \Gamma$ and $f_{L, R}=\frac{1}{2}(1 \mp f)$, so that $f \equiv f_{R}-f_{L} \in[0,1]$ $\left(f_{L} \leq f_{R}, 0 \leq f_{L}, f_{R} \leq 1\right)$ is the parameter controlling the driving strength. Small $f$ implies that the system is weakly driven by the external baths and behaves as in the linear response

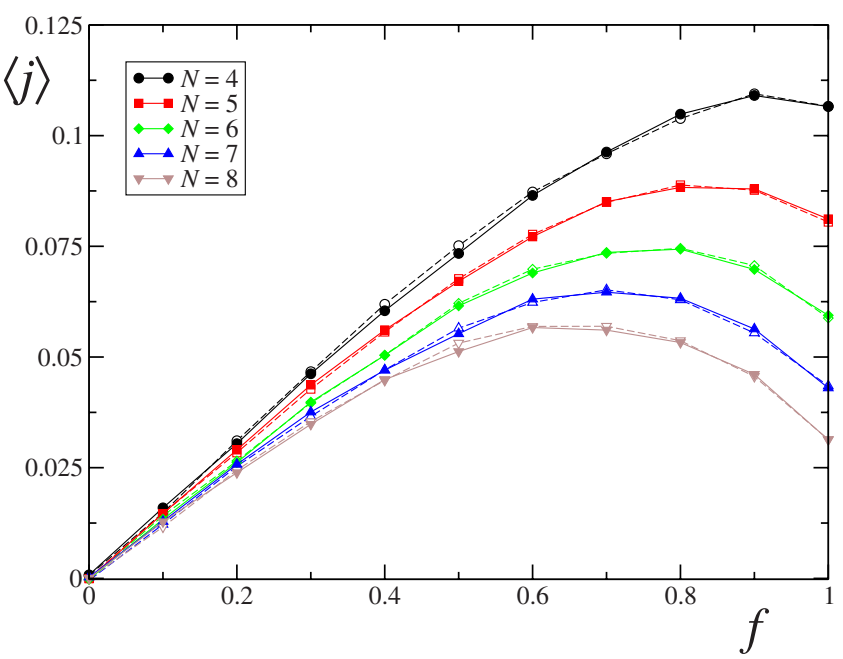

FIG. 2. (Color online) Spin current for the $\sigma$ (full curves and symbols) and the $\tau$ species (dashed curves and empty symbols) of spin as a function of the driving strength for the Hamiltonian in Eq. (14) with $U=5$. The system-bath coupling is set equal to $\Gamma=0.5$; the simulation time (QT approach) is $T=2 \times 10^{5}$. Note that curves and symbols for the $\sigma$ and $\tau$ species are nearly superimposed. For the Hubbard model we set $t=1$ as the system's energy scale.

regime. In the opposite limiting case $f=1$, the left (right) bath only induces up-down (down-up) spin flips for both spin species.

Using the method of quantum trajectories we evaluated the stationary spin currents $\left\langle j_{\sigma}\right\rangle,\left\langle j_{\tau}\right\rangle$ for the two species of spins. Due to the mapping between the electrons described by the Hamiltonian in Eq. (12) and the spins obeying Eq. (14), this spin current exactly equals the electronic current in the Hubbard model. In particular, $\left\langle j_{\sigma}\right\rangle\left(\left\langle j_{\tau}\right\rangle\right)$ is the current flow of electrons with spins pointing up (down), which is the crucial physical quantity in charge transport.

Perhaps the most interesting result we found in the current behavior as a function of the driving is the emergence of a NDC phenomenon for sufficiently strong drivings, as shown in Fig. 2. It happens that, while for small $f$ values the current increases, there exists a value $f^{*}$ at which $\langle j\rangle$ exhibits a maximum and then, further increasing $f$, it decreases.

One can now question whether or not this nonmonotonic behavior is stable when varying the Hamiltonian parameters $t$ and $U$. In all simulations reported here we fixed energy units by setting $t=1$. In the limiting case where $U=0$, the fermions in the Hubbard model are noninteracting, therefore a linear regime in which the current is always proportional to the driving strength is expected. In view of these considerations, it is tempting to assume the existence of a critical value $U^{*}$ in the Hamiltonian parameter space separating the linear and NDC behaviors of the current. As a matter of fact, within numerically accessible system sizes, we observed NDC only for $U>U^{*}$, where $U^{*} \approx 2$. This can be seen from Fig. 3, where we plot the maximal current drop, measured by $\langle j\rangle_{f=f^{*}}-\langle j\rangle_{f=1}$, as a function of the on-site interaction strength $U$. From the inset it is clear that, while for $U \ll 2$ the current is proportional to the driving, for $U \gg 2$ a bell-shaped behavior emerges. Of course, on the basis of the data presented in Fig. 3 one cannot exclude that $U^{\star}$ drops with $N$. In this 


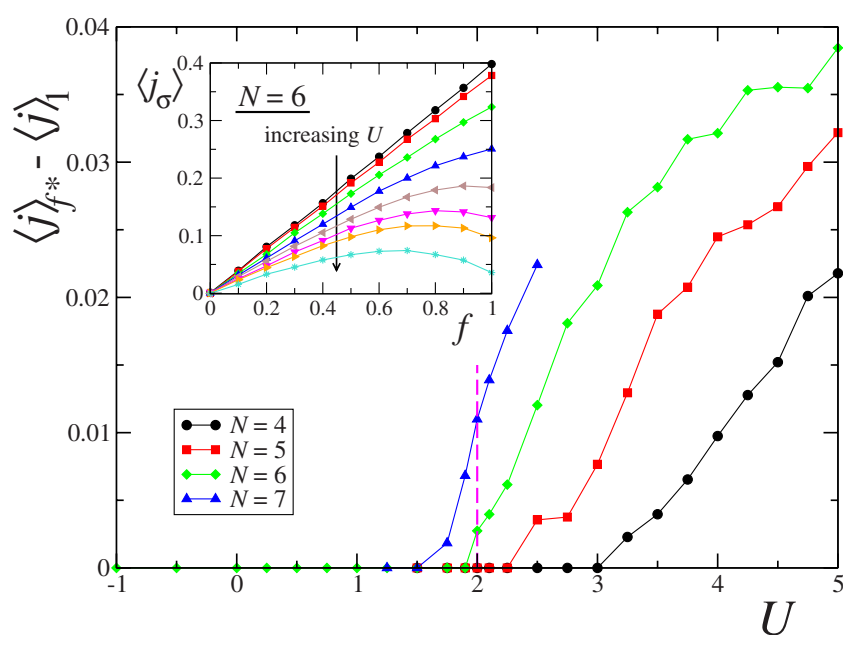

FIG. 3. (Color online) Maximum current minus current at maximum driving strength, $\langle j\rangle_{f^{*}}-\langle j\rangle_{1}$, as a function of the on-site repulsion $U$. In the inset we show the spin current as a function of $f$ for a fixed size $N=6$ and different values of $U$ : from top to bottom $U$ $=0$ (circles), 0.5 (squares), 1 (diamonds), 1.5 (triangles up), 2 (triangles left), 2.5 (triangles down), 3 (triangles right), and 5 (stars). Data are for $\Gamma=1$, and the simulation time (QT approach) is $T$ $=10^{5}$. The current is plotted only for the $\sigma$-spin species; differences with the $\tau$-spin species are negligible in the scales of the figure.

scenario, in the thermodynamic limit NDC would be observed for any $U>0$; nonetheless, we point out that a meanfield qualitative argument given at the end of Sec. V D supports the existence of NDC for $U \geqslant 2$, thus agreeing with our findings in Fig. 3. In any case, a significant result of our numerical simulations is the emergence of NDC in a physically relevant transport model such as the Hubbard model at small system sizes $N \geq 4$.

\section{SPINLESS FERMION MODEL}

The investigation of the far-from-equilibrium properties of the Hubbard model is numerically demanding and an analytical treatment appears difficult. Therefore, in what follows we will focus on a simplified model, usually referred to as the $t-V$ model, where the spin degree of freedom is neglected. This model is numerically much more convenient. Moreover, it will help us in gaining a deeper understanding of the peculiarities of charge transport discussed in Sec. III for the Hubbard model.

The $t-V$ model considers spinless fermions instead of spin-1/2 particles. Its Hamiltonian reads as follows:

$$
\mathcal{H}_{S}=-t \sum_{j}\left(c_{j}^{\dagger} c_{j+1}+c_{j+1}^{\dagger} c_{j}\right)+V \sum_{j} n_{j} n_{j+1} .
$$

Similarly to Hubbard model (12), the operators $c_{j}^{\dagger}, c_{j}$ create and annihilate a spinless fermion at site $j=1, \ldots, N$ (therefore they satisfy canonical anticommutation rules), while $n_{j}=c_{j}^{\dagger} c_{j}$ is the corresponding number operator. The system parameters $t$ and $V$ describe, respectively, the nearestneighbor hopping strength and the fermionic repulsion between contiguous sites.
In direct analogy with what has been discussed for the Hubbard model, we take open boundary conditions and couple both ends of the chain to some external baths that inject and extract fermions. Now the number of Lindblad operators is halved since we removed the spin degree of freedom,

$$
\begin{aligned}
& L_{1}=\sqrt{\Gamma_{L} f_{L}} c_{1}^{\dagger}, \quad L_{2}=\sqrt{\Gamma_{L}\left(1-f_{L}\right)} c_{1}, \\
& L_{3}=\sqrt{\Gamma_{R} f_{R}} c_{N}^{\dagger}, \quad L_{4}=\sqrt{\Gamma_{R}\left(1-f_{R}\right)} c_{N} .
\end{aligned}
$$

The parameters $\Gamma_{L}, \Gamma_{R}, f_{L}$, and $f_{R}$ play roles analogous to those of the corresponding parameters introduced for spinful fermions in Eqs. (5) and (6).

The Hamiltonian in Eq. (16) can be mapped into an XXZ Heisenberg spin chain plus some spurious contributions, consisting in external transverse magnetic fields, which are irrelevant in the isolated (Hamiltonian) case and do not qualitatively modify the spin current behavior (see Appendix B). Therefore we shall neglect these spurious terms and concentrate on the XXZ spin-1/2 system whose autonomous Hamiltonian is given by

$$
\mathcal{H}_{S}=\sum_{j=1}^{N-1}\left[J_{x}\left(\sigma_{j}^{x} \sigma_{j+1}^{x}+\sigma_{j}^{y} \sigma_{j+1}^{y}\right)+J_{z} \sigma_{j}^{z} \sigma_{j+1}^{z}\right],
$$

where $\sigma_{j}^{\alpha}(\alpha=x, y, z)$ are the Pauli matrices of the $j$ th spin and $\Delta \equiv J_{z} / J_{x}$ denotes the $x z$ anisotropy; $N$ is the total number of spins. In the $t-V$ model language of Eq. (16), the couplings in Eq. (18) are given by $J_{x}=-t / 2$ and $J_{z}=V / 4$, so that the anisotropy $\Delta=-V / 2 t$. Strictly speaking, since fermionic interaction between contiguous sites is repulsive $(V>0)$, this would correspond to antiferromagnetic transverse couplings $J_{z}>0$. Nonetheless, when considering XXZ spin model (18), one is not a priori forced by this constraint and can also analyze the ferromagnetic case $J_{z}<0$. Hereafter we set $J_{x}=1$ as the system's energy scale.

In the spin-1/2 picture, Lindblad operators (17) are mapped into operators flipping the border spins. Indeed as explained in Appendix B, apart from uninfluent phase factors, we have $c_{j}^{\dagger} \rightarrow \sigma_{j}^{+}$and $c_{j} \rightarrow \sigma_{j}^{-}$. The Fermi function $f_{L, R}$ is such that $2 f_{L, R}-1 \in[-1,1]$ is the corresponding bath's magnetization per spin in dimensionless units. As we did for the Hubbard model, we choose (with the exception of Sec. V B 4) a symmetric driving: $\Gamma_{L}=\Gamma_{R} \equiv \Gamma$ and $f_{L, R}=\frac{1}{2}(1 \mp f)$, so that $f \equiv f_{R}-f_{L} \in[0,1]\left(f_{L} \leq f_{R}, 0 \leq f_{L}, f_{R} \leq 1\right)$ is a single parameter controlling the driving strength. When $f$ is small we are in the linear response regime, while in the limiting case $f=1$ (corresponding to $f_{L}=0, f_{R}=1$ ) the left (right) bath only induces up-down (down-up) spin flips. The spin current is computed as in Eq. (15) but without the contribution of the $\tau$ species,

$$
j=J_{x}\left(\sigma_{k}^{x} \sigma_{k+1}^{y}-\sigma_{k}^{y} \sigma_{k+1}^{x}\right) .
$$

Quantitative numerical and semianalytical analyses of model (18) are easier than those of model (14). In particular, the local Hilbert space is halved: for a fixed number of sites $N$, the size $\mathcal{N}=2^{N}$ of a generic state vector describing the system is decreased by a square root factor with respect to the size $\left(2^{N}\right)^{2}$ of a state vector for spin ladder (14) of length 
$N$. It is therefore clear that, without truncating the Hilbert space, using the Monte Carlo wave function method one is able to simulate chains of twice the length of a ladder with the same computational cost. We wish to note that in the spin ladder systems we have so far used only the QT method to perform numerical simulations. Also the MPO approach could, in principle, be used by simply joining two sites from the opposite spin chains into a single site with a local dimension of 16 (for a single chain it is 4 ). The complexity of time evolution increases by a factor $4^{3}$ at a fixed matrix dimension $D$ (see Appendix A for definitions) because of singular value decompositions of $16 D \times 16 D$ matrices instead of $4 D \times 4 D$.

\section{SPIN TRANSPORT PROPERTIES IN A HEISENBERG CHAIN}

In this section, we study the far-from-equilibrium transport properties of XXZ Heisenberg spin chain (18), with the two edge spins coupled to external baths, as described in Sec. IV. We first present, in Secs. V A-V C, the results of our numerical simulations, focusing on the NDC phenomenon and on the appearance of a long-range spin ordering into ferromagnetic domains. Then, in Sec. V D, we qualitatively explain our results in terms of one-magnon localization. Since our findings are suggestive of a phase transition with the emergence of long-range order, we have also searched for numerical evidence of such transition by analyzing the spinspin correlation function. Our data displayed in Appendix C, even if not conclusive, show a dramatic slowing down of the correlation decay in the NDC regime even though the accessible system sizes are too small for a quantitative analysis of a possible phase transition. Finally, in Sec. V E we rephrase the results in terms of the fermionic current.

Note that the spin current in the XXZ model with antiferromagnetic coupling is the same as the charge current in the $t$ - $V$ model; therefore all the results we discuss here for $J_{z}>0$ also apply to the case of fermionic transport in Eq. (16). For the spin transport we also considered cases where $J_{z}<0$, thus corresponding to a rather unphysical attractive fermionic interaction in the $t$ - $V$ model; quite surprisingly, we found that data obtained for the XXZ chain are insensitive to the sign of the transverse coupling $J_{z}$.

As far as we know, transport properties of the autonomous model described by Eq. (18) have been extensively analyzed only within the linear response regime ${ }^{16-19}$ even if a fully comprehensive understanding is still lacking. In particular, it has been found that the low-temperature and the hightemperature thermodynamic transport properties are essentially determined by the $x z$ anisotropy $\Delta .{ }^{16}$ In the zero magnetization sector, the $\mathrm{XXZ}$ model is an ideal conductor for $|\Delta|<1$, while numerical data ${ }^{17}$ suggest that the system is a normal (diffusive) spin conductor for $|\Delta|>1$. The normal conduction in the $|\Delta|>1$ regime has been recently confirmed for systems of much larger size, ${ }^{33} N \sim 100$ (see also Ref. 35). The above two distinct behaviors may be associated to two different system phases at zero temperature: for $-1 \leq \Delta \leq 1$ the system is gapless, while for $\Delta<-1(\Delta>1)$ it is ferromagnetically (antiferromagnetically) ordered, and the ground state exhibits a finite gap with the first excited state.

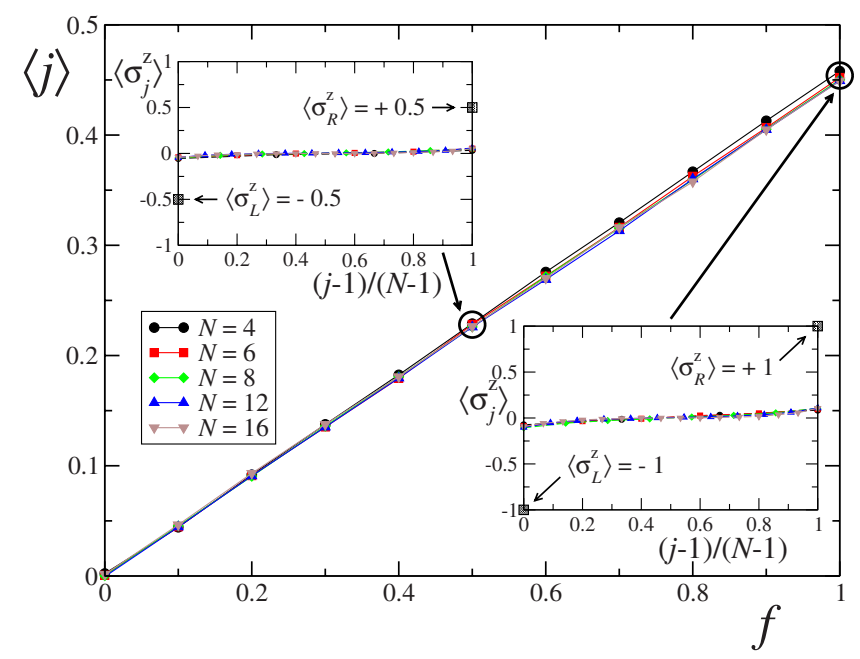

FIG. 4. (Color online) Spin current as a function of the driving strength for $\Delta=0.5$. The system-bath coupling is set equal to $\Gamma=1$. The insets show spin magnetization profiles versus the scaled spin index coordinate for two values: $f=0.5,1$. Data are obtained from the QT approach.

We now investigate the far-from-equilibrium properties of the XXZ Heisenberg spin chain beyond the linear response regime.

\section{A. Gapless regime}

We start by considering the gapless phase, where the linear response theory predicts ballistic transport. We found that in the regime $|\Delta|<1$ the current is always proportional to the driving and independent of the chain length $N$; this holds for any value of the driving strength $f \in[0,1]$. Remarkably, there are no appreciable quantitative differences between the current evaluated with a ferromagnetic $\left(J_{z}<0\right)$ or antiferromagnetic $\left(J_{z}>0\right)$ coupling. Figure 4 displays the spin current as a function of the driving strength $f$ for $\Delta=0.5$. The corresponding spin magnetization profiles for two distinct values of $f$ are displayed in the insets as a function of the site index. In both cases we can see a nearly flat profile, which is typical of systems with ballistic spin propagation; ${ }^{53}$ most importantly, we notice that the stationary spin magnetization at the borders is very different from the bath magnetizations $\left\langle\sigma_{L, R}^{z}\right\rangle=\mp f$.

\section{B. Gapped regime}

The transport properties are much more interesting in the gapped phase where, in the linear response regime $f \ll 1$, numerical data suggest normal diffusive transport. ${ }^{17,33}$ For $|\Delta|>1$ the spin current is no longer monotonic with $f$ and exhibits a typical bell-shaped behavior, as we observed for the Hubbard model (see Fig. 2).

In Fig. 5 we plot the spin current as a function of $f$. For small $f$ the system behaves as a normal Ohmic conductor, as expected from linear response results: ${ }^{17,33}$ namely, the current increases like $\langle j\rangle \propto f / N$. After a given value $f^{*}$ of the driving at which the current reaches its maximum, it starts decreasing with $f$ until it is strongly suppressed at $f=1$. De- 


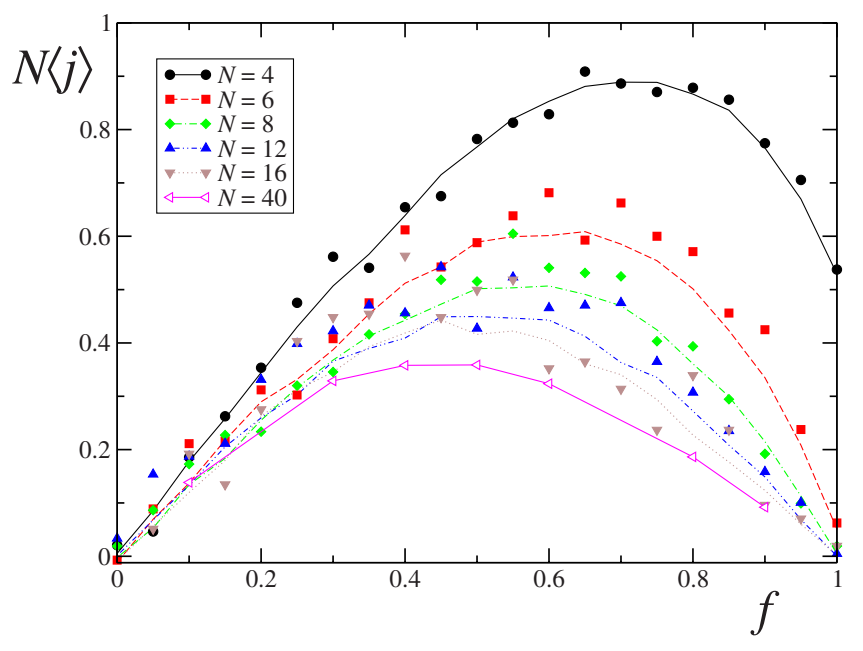

FIG. 5. (Color online) Spin current as a function of the driving strength $f$ in a chain with anisotropy $\Delta=2$. The system-bath coupling is set equal to $\Gamma=4$. Symbols for $N \leq 16$ are obtained using QT after a simulation time $T=2.5 \times 10^{3}$, while curves display data for a longer integration time $T=7.5 \times 10^{4}$. Data for $N=40$ are obtained by MPO, with integration times up to $T=4000$. Long-time data for $N \leq 16$ are quoted from Ref. 32 .

tails on the scaling of $\langle j\rangle$ with the system size at $f=1$ are given in Sec. V B 1. Here we just point out that, interestingly, NDC is already visible after short integration times: as a matter of fact, with the QT approach (see data for $N \leq 16$ ), the characteristic bell-shaped behaviors can be obtained after a simulation time $T \sim 2.5 \times 10^{3}$ (symbols) that is much shorter than the one required to reach the stationary values, $T \sim 10^{5}$ (curves); furthermore, NDC features are present even after very short times, $T \sim 5 \times 10^{2}$.

The data shown in Fig. 5 are suggestive of a transition from a normal spin conductor phase at small $f$ to an insulator phase at large $f$. On the other hand, for the achievable system sizes, the value $f^{\star}(N)$ where the current reaches a maximum drops with $N$. Therefore, in principle, we cannot exclude an alternative scenario where at the thermodynamic limit the system becomes an insulator at any driving strength $f$. In any case, as far as the bias $f$ is increased, a substantial modification of spin transport properties becomes apparent. These results are suggestive of a far-from-equilibrium quantum phase transition. In the light of a recent paper of one of us, ${ }^{22}$ we have analyzed the spin-spin correlation functions in order to see the possible emergence of a phase transition that should be characterized by the emergence at strong driving strength $f$ of a long-range correlation order. Numerical data, even if not conclusive, are shown in Appendix $\mathrm{C}$ and are supported by such a behavior.

\section{Behavior at $f=1$}

As hinted in Ref. 32, in order to understand the physical mechanism lying at the basis of NDC, we have to analyze the stationary spin magnetization profiles. These are shown in Fig. 6. Note that, in contrast with the fast-time raising up of the NDC phenomenon, a much longer integration time is required in order to reach a good convergence for the spin

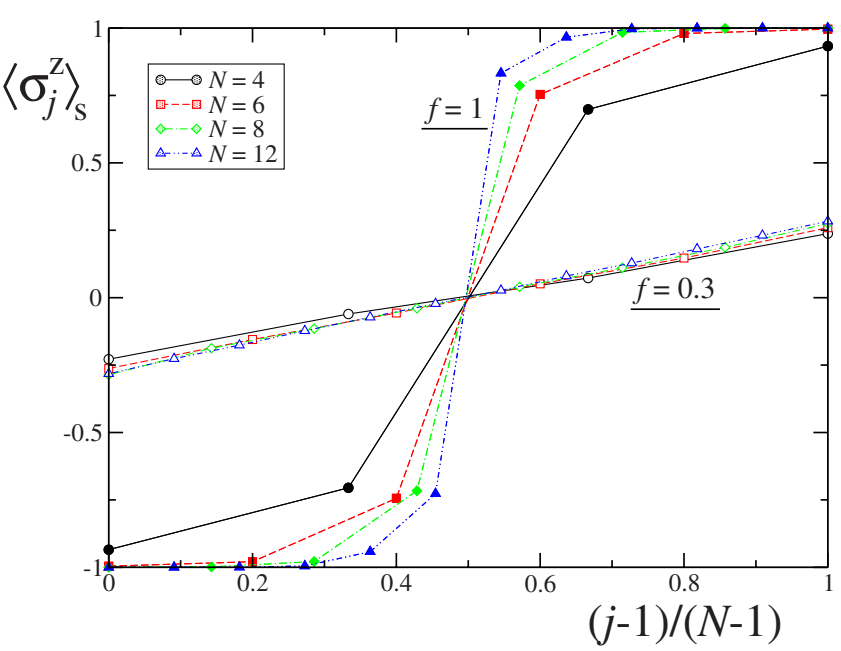

FIG. 6. (Color online) Spin magnetization profiles versus scaled spin index coordinate for the same parameters as in Fig. 5, and driving strengths $f=0.3$ (empty symbols) and $f=1$ (filled symbols). Data are obtained from the QT approach. (Figure acknowledged from Ref. 32.)

magnetizations due to the equilibration time scales that, at $f=1$, grow exponentially with the distance of the spin from the chain border. ${ }^{32}$

As shown in Fig. 6, magnetization profiles in the linear response regime $f \ll 1$ exhibit a constant linear gradient, where the magnetizations of the two edge spins are close to the bath magnetizations $\mp f$, as it is expected for normal Ohmic conductors. In the limiting case $f=1$ a peculiar stationary state characterized by two ferromagnetic domains that are oppositely polarized appears. Moreover their relative width increases with the system size. These domains are eventually responsible for strongly suppressing the spin current since they inhibit spin flips. Strictly speaking, evidence of the formation of such domains is also visible for smaller though strong drivings, where the NDC effect is established (see Sec. V B 2). We will explain later in Sec. V D the physical mechanism leading to the formation of such domains in the gapped phase. Here we stress that, as for the gapless regime, we found no quantitative differences between a ferromagnetic and a antiferromagnetic coupling. This may be somewhat counterintuitive since in this last case ferromagnetic domains correspond to a highly excited state for the autonomous system; the mechanism therefore has its roots in the genuine far-from-equilibrium dynamics.

We already observed that the current for small driving strengths behaves as a normal Ohmic conductor: $\langle j\rangle \propto f / N$. On the other hand, Fig. 7 shows that at maximum bias $f=1$ the current drops to zero exponentially with $N$, that is, the system is an insulator. Unfortunately we could not achieve system sizes larger than $N=12$ since there the current is very small, therefore it would require a huge number of time steps in order to get reliable results, thus making simulations unfeasible (already for $N=12$ the simulation time $T=7.5 \times 10^{4}$ is not long enough: the last point in the figure has been obtained by observing only one spin flip during the whole QT simulation). 


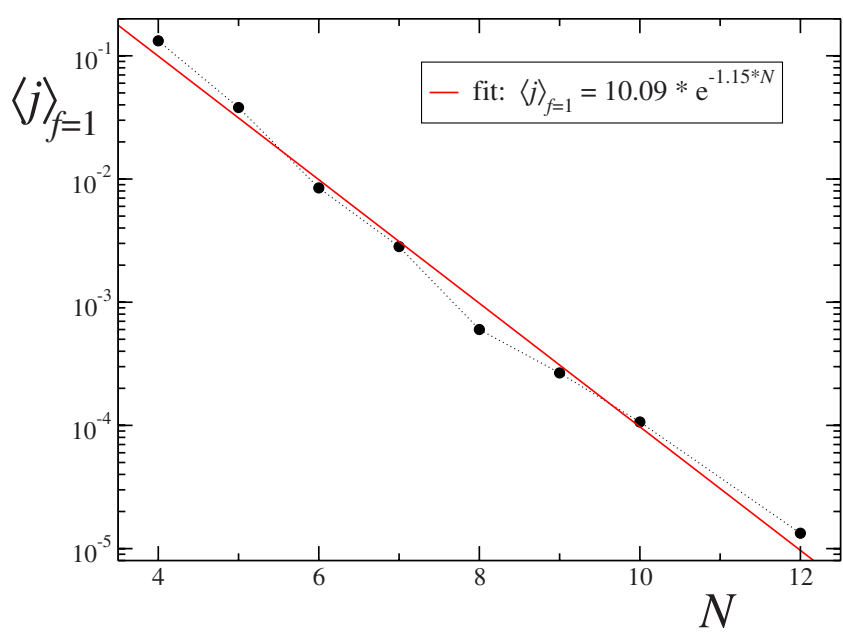

FIG. 7. (Color online) Spin current at a fixed driving strength $f=1$ for the Heisenberg spin model with $\Delta=2$. The system-bath coupling is set equal to $\Gamma=4$; the simulation time (QT approach) is $T=7.5 \times 10^{4}$. The thick curve displays an exponential fit of numerical data (circles).

\section{Spin blockade}

At maximum driving the current is strongly suppressed due to the fact that ferromagnetic domains of macroscopic length $\sim N / 2$ are formed. Nonetheless, a strong inhibition of the spin current can be also achieved by only creating a much smaller ferromagnetic region close to each bath. Indeed, signatures of this spin blockade mechanism are already seen at $f \sim 0.9-0.95$, where asymptotically only a couple of outer spins reach magnetization values close to \pm 1 , but still the current is far below its maximum value $\langle j\rangle_{f^{\star}}$.

Some spin magnetization profiles for strong drivings are explicitly shown in Fig. 8. The dotted-dashed curve corresponds to a maximum driving; there macroscopic ferromag-

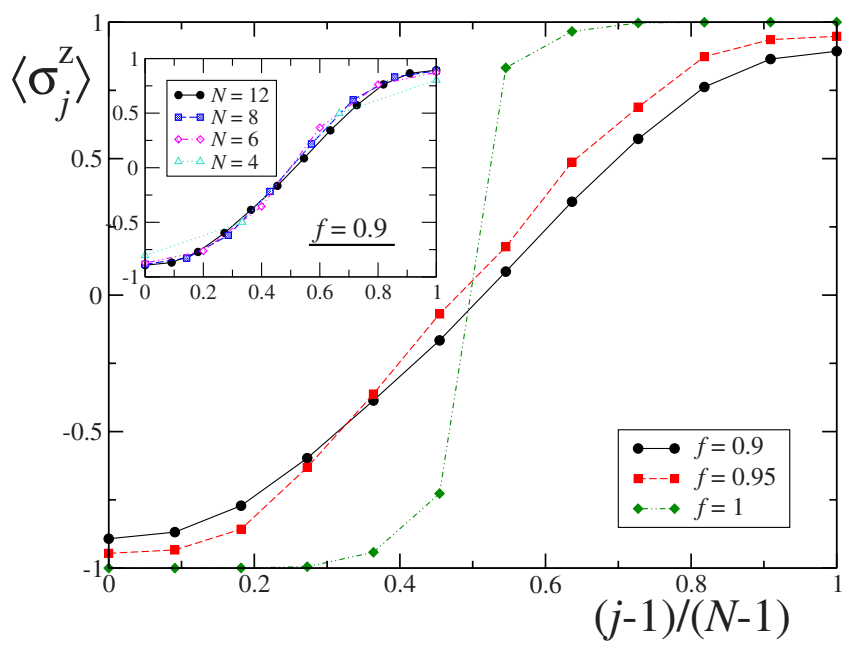

FIG. 8. (Color online) Spin magnetization profiles versus scaled spin index coordinate in the insulating case $\Delta=2$ with a system-bath coupling $\Gamma=4$ for strong drivings. In the main panel we fix a system size $N=12$ and choose different values of $f$, while in the inset we focus on the case $f=0.9$ and vary the size according to the legend. Data are obtained from the QT approach.

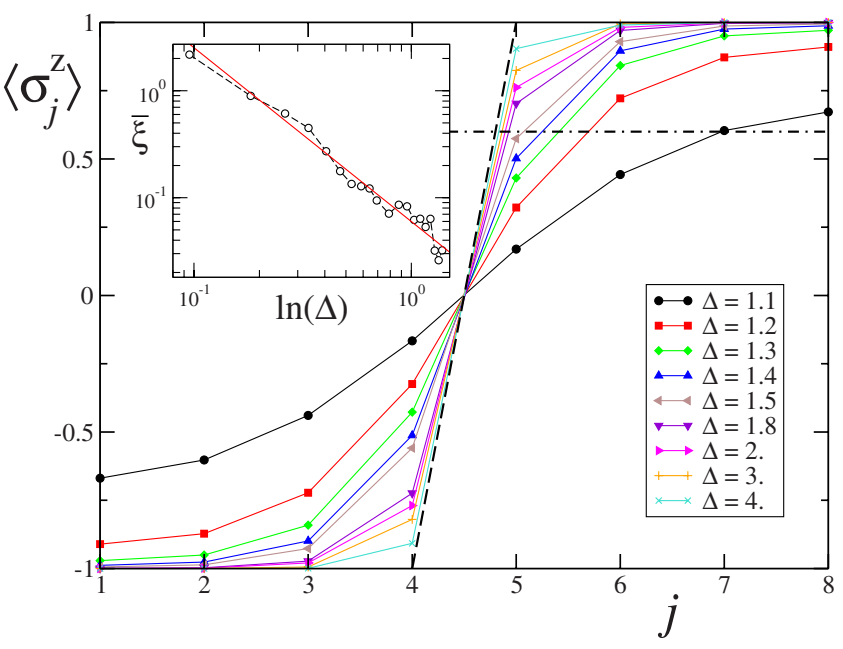

FIG. 9. (Color online) Spin magnetization profiles versus scaled spin index coordinate for a chain with $N=8$ spins at maximum driving $(f=1)$ for different values of $\Delta>1$; the system-bath coupling is set equal to $\Gamma=1$. In the inset we plot an estimate of the thickness of the interface region $\bar{\xi}$ as a function of $\ln (\Delta)$; the straight line shows the fit $\bar{\xi} \propto(\ln \Delta)^{-1.625}$. Data are obtained from the QT approach.

netic domains are clearly visible. The other ones are for $f$ slightly less than 1 , but it is still possible to see that a couple of spins close to the borders are nearly perfectly down or up polarized. In the inset we fix $f=0.9$ and vary the system size; we notice that, when increasing $N$, the number of spins involved in the spin blockade also increases, in accordance with the results previously shown for $f=1$.

\section{Thickness of the interface region}

An interesting point that can be addressed is the analysis, at maximum driving strength $f=1$, of the characteristic thickness $\xi$ of the interface region, located around the chain center and dividing the two ferromagnetic regions. According to our data shown in Fig. 9, this size depends on the system anisotropy $\Delta$. On the other hand, we checked that dependence on $N$ is negligible. In order to give a rough estimate of $\xi$, we fixed a threshold $\overline{\left\langle\sigma_{j}^{z}\right\rangle}$ (horizontal dotted-dashed line in the figure, corresponding to $\left.\overline{\left\langle\sigma_{j}^{z}\right\rangle}=0.6\right)$ and then evaluated the distance $\bar{\xi}$ of the point in each spin magnetization profile reaching that value from the limiting case $(\Delta \rightarrow \infty)$ in which the chain is exactly split into two ferromagnetic domains (due to the fact that our problem is on a lattice of finite length, the $\Delta \rightarrow \infty$ magnetization for $N / 2<j<N / 2+1$ is estimated after joining the two perfectly ferromagnetic domains with a skew dashed line). In the inset we study the dependence of the distance $\bar{\xi}$ on $\Delta$; the straight line shows the fit $\bar{\xi} \propto(\ln \Delta)^{-1.625}$. We point out that the semianalytical argument of the one-magnon-localization length that will be discussed in Sec. V D predicts that the size $\xi$ of the interface region is of the order of the one-magnon-localization length, namely, logarithmic in $\Delta$ and $N$ independent.

\section{Stability of the ferromagnetic domains}

Until now we have only considered situations in which system-reservoir couplings $\Gamma_{L, R}$ and driving strengths $\mu_{L, R}$ 

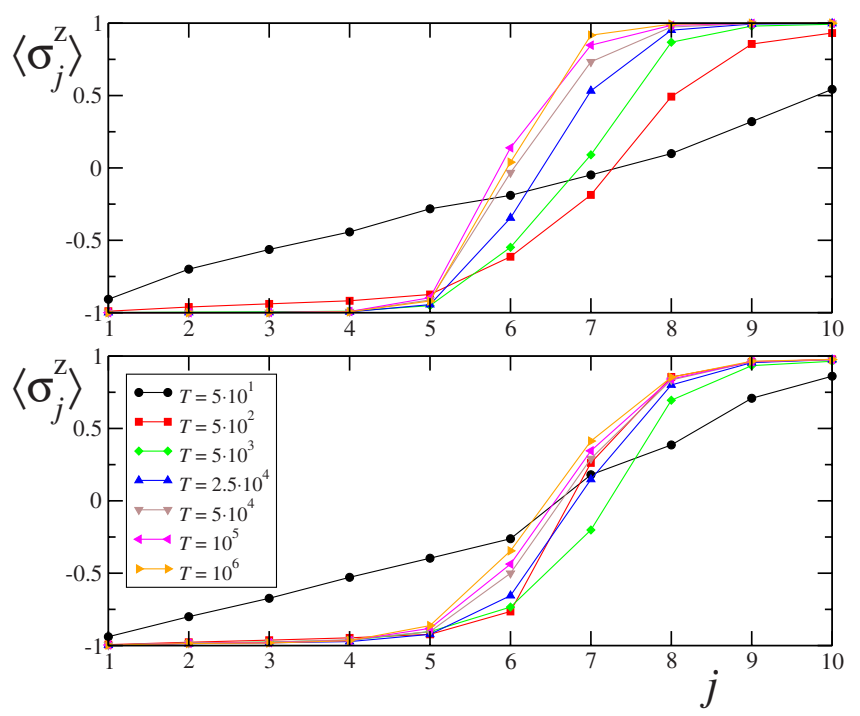

FIG. 10. (Color online) Spin magnetization profiles versus spin index coordinate for a chain with $N=10$ spins and anisotropy $\Delta$ $=2$ at different integration times $T$. Top: symmetric drivings such that $f=1$ and system-bath couplings $\Gamma_{L}=1, \Gamma_{R}=0.1$. Bottom: the system-bath coupling for the left and the right baths is kept fixed and equal to $\Gamma=1$, while the drivings are chosen asymmetrically as $f_{L}=0, f_{R}=0.99$. Data are obtained from the QT approach.

are symmetric. At this step one may wonder if the position of the domain wall between the two ferromagnetic regions in the spin magnetization profiles is stable against the breaking of such symmetry. Any imbalance, though small, may, in principle, cause a shift of the interface region toward one of the boundaries of the chain. This would raise some doubts about the stability of the previously depicted scenario, making our discussion relevant only for fine-tuned values of the Lindblad parameters. Below we show that this is not the case.

Imbalances of the couplings $\Gamma_{L, R}$ have quite tiny effects on the steady state at maximum driving, as one can see from the upper panel of Fig. 10. We put there a strong asymmetry by setting $\Gamma_{L}=1, \Gamma_{R}=0.1$ and thus admitting a coupling to the right bath that is one order of magnitude smaller than the one to the left bath. Differences with respect to the symmetric case are apparent for finite integration times $T$, where the domain wall is clearly shifted to the right. On the other hand, as far as $T$ is increased, profiles become more symmetric. It is not a priori clear whether the steady state is perfectly symmetric, as those in Fig. 6: from our data we cannot rule out possible deviations in the position of the domain wall, which are logarithmic in the coupling imbalance; this would be hardly detectable from a merely numerical analysis.

Stronger modifications are induced by imbalances on the driving strengths $\mu_{L, R}$. Indeed, in that case even a small asymmetry would cause a weaker spin blockade on one side, as discussed in Sec. V B 2 (see the lower panel of Fig. 10, in which we put $f_{L}=0, f_{R}=0.99$ ); as a consequence, a deformation and a broadening of the interface region are also established. Nonetheless, we point out that these modifications appear to be continuous in the degree of imbalance and thus, in principle, controllable.

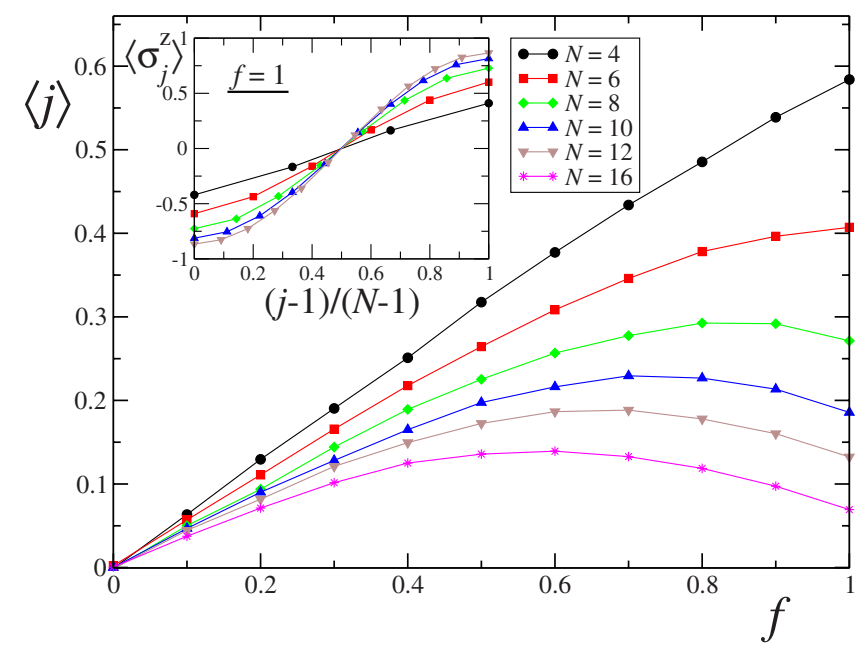

FIG. 11. (Color online) Spin current as a function of the driving strength for the isotropic case $\Delta=1$. The system-bath coupling is set equal to $\Gamma=2$. The simulation time (QT approach) is $T=5 \times 10^{4}$. In the inset we show the spin magnetization profiles versus scaled spin index coordinate at maximum driving $f=1$. Different curves stand for various system sizes.

\section{XXX Heisenberg isotropic model}

The isotropic XXX Heisenberg chain (that is, $|\Delta|=1$ ) corresponds to a limiting, nonetheless interesting situation, since molecular compounds that are used to investigate onedimensional spin-1/2 transport properties are often very well described by isotropic antiferromagnetic Heisenberg exchange couplings. ${ }^{39,54}$

In Fig. 11 we show some numerical data concerning the behavior of the spin current with respect to the driving field: NDC is visible only for sufficiently long chains $(N \geq 8)$. A qualitative understanding of this result comes from an analysis of the spin magnetization profiles at $f=1$, which are plotted in the inset. As a matter of fact, we can recognize a situation that is similar to the one already observed at $|\Delta|>1$ and $f \lesssim 1$ (see Fig. 8): by increasing $N$, a partial spin blockade of the outermost spins is established. This progressively inhibits the current flow along the chain, thus setting up the NDC mechanism. Notice also that, at small $N$ values $(N=4,6)$, the spin blockade is very weak; this prevents systems of very small size from exhibiting the NDC phenomenon even though a nonlinear dependence of the spin current on the driving strength can already be seen.

We conjecture that also for the isotropic XXX chain the NDC phenomenon is stable at the thermodynamic limit. Indeed, our simulations suggest the following picture: on one hand, at small $f$ the current decreases as $\langle j\rangle \propto N^{-\alpha}$ with $\alpha \approx 0.4$ (data for $f=0.2$ are shown in Fig. 12, left; we checked that this is consistent for all $f \leqq 0.3$ ). On the other hand, at $f=1$ it drops to zero faster than linearly with $N$ (probably exponentially, as suggested by Fig. 12, right), thus indicating a relative current drop $1-\langle j\rangle_{f=1} /\langle j\rangle_{f=f^{*}}$ that increases with $N$ toward the unit value; here $f^{*}$ denotes the driving at which the current is maximal. In both panels a log-log scale has been used so to make visible the distinction between a power-law scaling at small $f$ and an exponential 

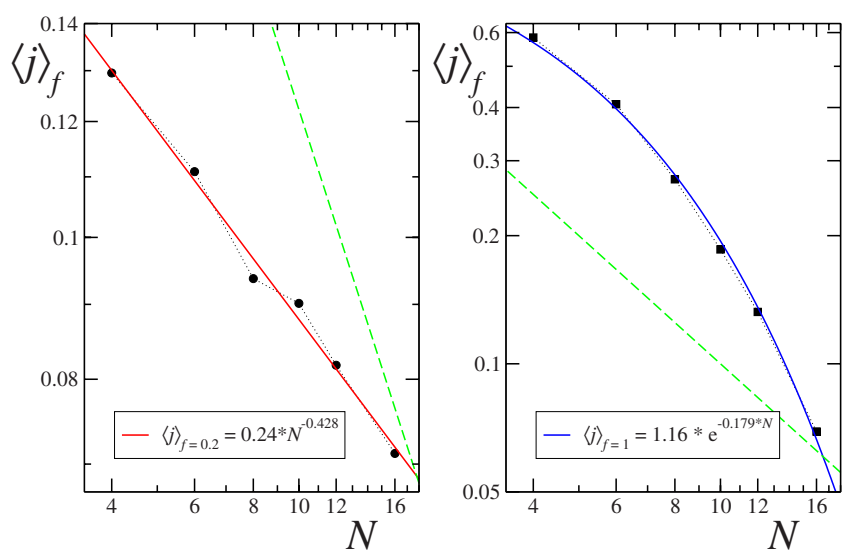

FIG. 12. (Color online) Spin current for $\Delta=1$ at a fixed driving strength, $f=0.2$ on the left, while $f=1$ on the right (see Fig. 11). Full curves are fits of numerical data (obtained from QT): while an inverse power-law fit works well at $f \ll 1$, for maximum driving an exponential fit seems adequate. The dashed lines indicate a behavior $\langle j\rangle_{f} \sim 1 / N$ and are plotted as guidelines.

behavior at $f=1$. We also plotted the $1 / N$ behavior (dashed lines) expected for normal Ohmic conductors to show that for $f \ll 1$ the decay is slower than that and for $f=1$ it is faster.

\section{One-magnon localization}

The formation of ferromagnetic domains and the negative differential conductivity phenomenon in the gapped regime $|\Delta|>1$ can be qualitatively explained in terms of localization of one-magnon excitations created at the borders of a ferromagnetic domain. We should immediately point out that the following argument is independent of the ferromagnetic of antiferromagnetic coupling. This reflects into the fact that the steady state with ferromagnetic domains is completely driven by dynamical effects and not by the system ground state, being actually an antiferromagnet for $J_{z}>1$.

Given a ferromagnetic state $|0\rangle \equiv|\downarrow \downarrow \ldots \downarrow\rangle$, one-magnon excitations have the general form $\sum_{k=1}^{N} \alpha_{k}|k\rangle$, where $|k\rangle=\sigma_{k}^{+}|0\rangle$ describes the state with the $k$ th spin flipped. If the autonomous XXZ chain has open boundary conditions, there is an energy gap $2\left|J_{z}\right|$ between the states $|1\rangle$ and $|N\rangle$ (spin-flip excitations at the boundaries) and the states $|2\rangle,|3\rangle, \ldots,|N-1\rangle$. Indeed, we have

$$
\begin{gathered}
\left\langle 0\left|\mathcal{H}_{s}\right| 0\right\rangle=(N-1) J_{z}, \\
\left\langle 1\left|\mathcal{H}_{s}\right| 1\right\rangle=\left\langle N\left|\mathcal{H}_{s}\right| N\right\rangle=(N-3) J_{z}, \\
\left\langle 2\left|\mathcal{H}_{s}\right| 2\right\rangle=\cdots=\left\langle N-1\left|\mathcal{H}_{s}\right| N-1\right\rangle=(N-5) J_{z},
\end{gathered}
$$

where $\mathcal{H}_{s}$ is XXZ Hamiltonian (18). Only nearest-neighbor spin-flipped states are coupled and the coupling strength is $2\left|J_{x}\right|$,

$$
\left\langle k\left|\mathcal{H}_{s}\right| k+1\right\rangle=2 J_{x}, \quad k=1, \ldots, N-1 .
$$

As shown in Appendix D, the autonomous model defined by Eqs. (20) and (21) is exactly solvable in the limit of large $N$ and spin-flip excitations created at the borders of the chain remain exponentially localized when $\left|J_{z}\right| /\left|J_{x}\right|=|\Delta|>1$ over a localization length $\ell \sim 1 / \ln |\Delta|$.

We now consider the coupling to external baths. First, it is instructive to discuss the case in which the system is coupled to a single fully polarized reservoir, $f_{L}=0$. Regardless of the anisotropy $\Delta$, the stationary state is pure and ferromagnetic, namely, $|\downarrow \downarrow \ldots \downarrow\rangle \downarrow \downarrow \ldots \downarrow \mid$, since Hamiltonian (18) conserves the overall magnetization while at the left boundary of the chain only the lowering operator $L_{2} \propto \sigma_{1}^{-}$acts (note that the convergence to the stationary ferromagnetic state can be rigorously proven following Ref. 25). As shown in Fig. 13 (see circles), the time scale required for the convergence of the $j$ th spin to the equilibrium state $|\downarrow\rangle$ scales exponentially with $j$. Consider now an intermediate state with $m$ spins. To enlarge the ferromagnetic domain, one-magnon excitations should be propagated, through $\sigma_{j}^{x} \sigma_{j+1}^{x}$ and $\sigma_{j}^{y} \sigma_{j+1}^{y}$ exchange couplings of Hamiltonian (18), across the ferromagnetic domain to the left chain boundary. Suppose, for instance, that we have the leftmost $m$ spins down and the $(m+1)$ th spin up and that this excitation propagates to the left bath; then the bath can flip this spin down, thus ending up with a ferromagnetic domain with $m+1$ leftmost spins down. The crucial point is that the one-magnon propagation is exponentially localized at $|\Delta|>1$. Hence, exponentially long time scales $\propto \exp (j / \ell) \sim \exp (j \ln |\Delta|)$ are required to polarize the $j$ th spin.

Consider now two baths at $f=1$. Due to exponential onemagnon localization, essentially only the nearest bath is felt by the spins in the chain, with the exception of an interface region between the two ferromagnetic domains, whose length is of the order of the one-magnon localization length. As shown in Fig. 13 (squares), apart from the interface region, the spin magnetization profiles for the spins closer to the left than to the right bath essentially evolve as in the single-bath case.

These ferromagnetic domains are responsible for strongly inhibiting spin flips and therefore for suppressing the spin current at $f=1$. Since at small $f$ the current grows linearly, we can conclude that, due to the continuity of $\langle j\rangle_{f}$, a region of negative differential conductivity exists. Finally, we note that, in agreement with our numerical data, the one-magnon argument does not distinguish between ferromagnetic $\left(J_{z}<0\right)$ and antiferromagnetic $\left(J_{z}>0\right)$ spin couplings.

The argument developed in this section can be extended to spin ladder model (14) obtained by applying a JordanWigner transformation (JWT) to the Hubbard Hamiltonian. Assume that we have a ferromagnetic domain of the $m$ spins of both species ( $\sigma$ and $\tau$ ) closest to, say, the left bath. Now consider a spin flip for the $(m+1)$ th spin, for instance, of the $\sigma$ species and assume that the spins of the $\tau$ species can be treated within the mean-field approximation. The only energy term not constant for autonomous evolution (14) restricted to the one-magnon sector ( $\sigma$ species) is $\frac{U}{4} \sum_{j} \sigma_{j}^{z} \tau_{j}^{z}$. Within mean-field approximation, we substitute $\tau_{j}^{z} \rightarrow\left\langle\tau_{j}^{z}\right\rangle$, and $\left\langle\tau_{j}^{z}\right\rangle=1$ for $i=1, \ldots, m$, while $\left\langle\tau_{m+1}^{z}\right\rangle=0$. There is an energy gap $\frac{|U|}{2}$ between states with the spin flipped belonging to sites from 1 to $m$ and the state with the $(m+1)$ th spin flipped. The hopping strength is $|t|$. Therefore magnon localization as in the XXZ model takes place for $|U|>2|t|$. Such prediction is compatible with the numerical results shown in Fig. 3, where NDC for the Hubbard model is observed only for 

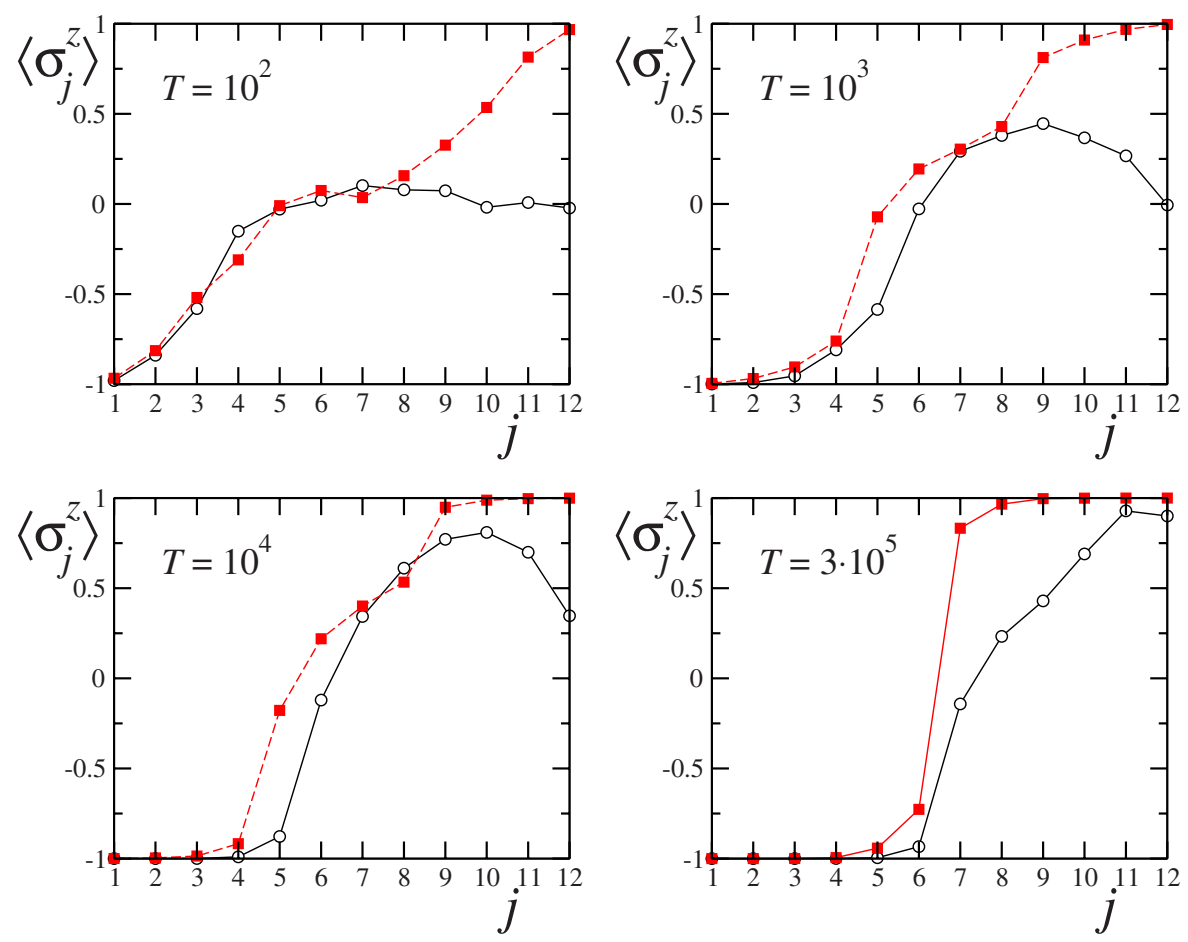

FIG. 13. (Color online) Spin magnetization profiles at $\Delta=2, \Gamma$ $=4$ for a maximal driving strength: $f=1$. The various panels correspond to different times $T$ (in QT simulation) at which the profiles are plotted. Circles are for a single bath coupled to the first $\operatorname{spin}(j=1)$, while squares are for two baths at the ends of the chain.

$U>U^{\star}$, with $U^{\star} \approx 2$. However, the argument is of a meanfield nature and therefore has to be considered weaker than the one developed for the XXZ chain.

\section{E. Discussion in terms of the charge current in the fermionic model}

At this stage, it is useful to summarize the main results obtained for the spin chain in terms of the original electronic $t$ - $V$ model. Since $\frac{1}{2}\left(1+\left\langle\sigma_{k}^{z}\right\rangle\right)$ corresponds to the electronic charge density at site $k$, the ferromagnetic domains observed at $f=1$ corresponds, in the fermionic picture, to a phase separation, with all the electrons frozen in the right half of the lattice close to the emitter electrode. Therefore, charge transport is inhibited, provided that $V / t>2$. Note that this charge clustering takes place in spite of the repulsive nature of electron-electron interactions. The magnon-localization argument can be straightforwardly reformulated in terms of the fermionic model. In particular, a one-magnon excitation becomes a single electron (hole) propagating on an empty (filled) lattice. The single-bath case can then be interpreted in terms of depletion (filling) of the lattice by means of a single lead, playing the role of a charge collector (emitter). This process requires exponentially long time scales at $V / t>2$. Finally, we point out that the NDC regime is observed for strongly interacting systems $(V / t>2)$ and in the far-fromequilibrium regime, corresponding to large bias voltages $e V \gg k_{B} T$, so that the Fermi functions for the collector and the emitter electrodes, evaluated at the energy differences $E_{1}$ and $E_{N}$, satisfy $f_{L} \approx 0$ and $f_{R} \approx 1$, respectively.

\section{NONINTEGRABLE MODEL: STAGGERED MAGNETIC FIELD}

The high-temperature transport properties in onedimensional quantum many-body systems are strongly af- fected by the presence of conservation laws..$^{20,21,55,56}$ In particular, the existence of local conserved quantities $Q_{n}, n$ $=1,2, \ldots$, typically leads to an ideally conducting-ballistic behavior at all the temperatures. This is a consequence of the inequality due to Mazur, ${ }^{57}$ which bounds the time averaged current-current autocorrelation function as $\quad \lim _{t \rightarrow \infty}(1 / t) \int_{0}^{t} d t^{\prime}\left\langle J\left(t^{\prime}\right) J(0)\right\rangle_{\beta} \geq \Sigma_{n}\left|\left\langle J Q_{n}\right\rangle_{\beta}\right|^{2}$, where $\langle X\rangle_{\beta} \equiv \operatorname{tr}[\exp (-\beta H) X] / \operatorname{tr}[\exp (-\beta H)]$ and $Q_{n}$ are chosen and normalized such that $\left\langle Q_{n} Q_{m}\right\rangle=\delta_{n m}$. As one can see, this argument essentially depends on, first, the existence of nontrivial conserved quantities (as is typically the case only for completely integrable systems) and, second, on the overlaps $\left\langle Q_{n} J\right\rangle_{\beta}$ between the conservation laws $Q_{n}$ and the transporting current $J$ in question. For example, for the XXZ model all the conservation laws have zero overlaps with the spin current, $\left\langle J_{s} Q_{n}\right\rangle=0$, which allows in the gapped regime $|\Delta|>1$ for the normal diffusive spin transport as discussed earlier, whereas, for example, the heat transport is ballistic as the energy current is just one of the conserved quantities $J_{E}=Q_{3}{ }^{55}$ However, even though the XXZ Heisenberg model is completely integrable, we have seen that the spin transport properties in the far-from-equilibrium regime are very different from the linear response regime behavior. Therefore, we might expect that the presence of NDC is not related to integrability of the Heisenberg model.

In order to check the stability of the nonlinear transport highlighted in the previous sections with respect to breaking system integrability, we considered a slightly modified spin chain model, in which a staggered magnetic field along the $z$ direction is added to the Heisenberg Hamiltonian of Eq. (18). Namely, we studied the following autonomous model:

$$
\mathcal{H}_{S}=\sum_{j=1}^{N-1}\left[\left(\sigma_{j}^{x} \sigma_{j+1}^{x}+\sigma_{j}^{y} \sigma_{j+1}^{y}\right)+\Delta \sigma_{j}^{z} \sigma_{j+1}^{z}\right]-B \sum_{j=1}^{N}(-1)^{j} \sigma_{j}^{z} .
$$




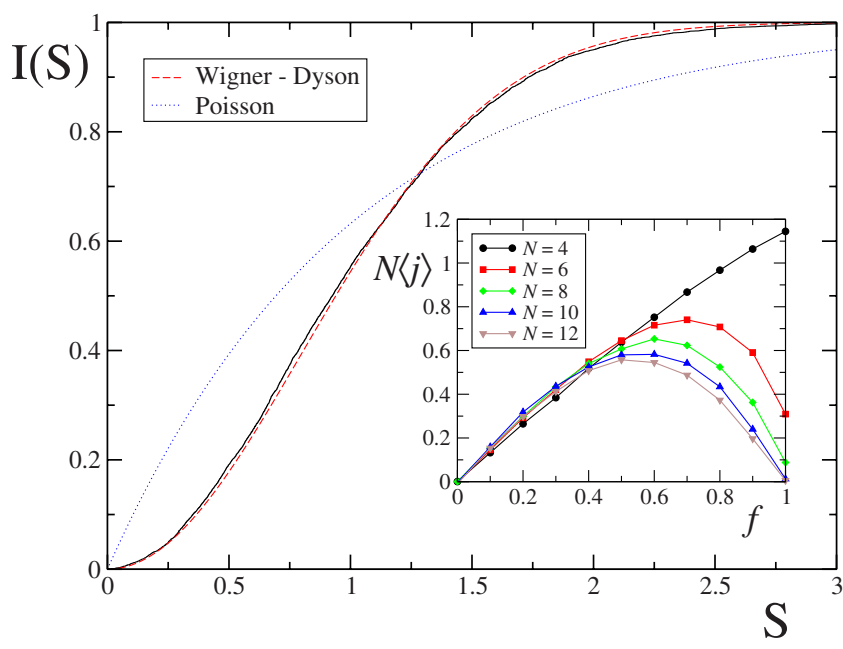

FIG. 14. (Color online) Integrated level spacing distribution $I(S)$ for the Hamiltonian in Eq. (22) with $\Delta=1.3, B=0.3, N=16$; data (full black curve) correspond to the zero magnetization sector. In order to apply the random matrix theory, the system Hamiltonian has to be diagonalized in a subspace in which no symmetries (except the time reversal) are left. For this purpose, in addition to fixing the total magnetization, we applied the staggered magnetic field on all spins except the first one [i.e., we supposed that the last sum in Eq. (22) runs from 2 to $N$ ]. This breaks the spatial reflection symmetry $j \rightarrow N-j$, without affecting the transport properties under investigation. Red dashed (blue dotted) curve indicates WignerDyson (Poissonian) statistics, typical for chaotic (integrable) systems. In the inset (data from QT) we plot the spin current as a function of the driving strength for different chain lengths; we fixed a system-bath coupling $\Gamma=1$.

Interestingly, model (22) exhibits a transition from integrability to quantum chaos when increasing the field strength $B$. This can be detected in the change in the spectral statistics of the system. ${ }^{58,59}$ In particular, in Fig. 14 we plot the integrated level spacing distribution $I(S)[I(S)$ is the probability that a randomly chosen level spacing-normalized to the mean level spacing -is less than $S]$. It is shown that, for a given set of parameters and $B \neq 0$, the level statistics follows the universal predictions of the random matrix theory (Wigner-Dyson statistics in the presence of time-reversal symmetry), as typical for chaotic (strongly nonintegrable) systems. In the inset we analyzed the corresponding spin current as a function of the driving strength: we found a qualitatively analogous behavior as in the integrable case, with a NDC regime still clearly visible. We also checked the presence of a normal Ohmic conduction for small drivings: from Fig. 15 one can see that, for $f=0.1$, the spin current scales as $\langle j\rangle \propto 1 / N$, according to Ohm's law of diffusive transport (see also Ref. 33); therefore spin transport is normally diffusive, as expected for a chaotic system. In analogy with the integrable case, for larger gradients where the current behaves highly nonlinearly, the spin current decays faster than linearly (at $f=0.5$ we found a decay $\langle j\rangle \sim \Delta S / N^{1.4}$, thus indicating an insulating behavior in the thermodynamic limit). Also magnetization profiles in that case are not linear anymore and the emergence of a weak spin blockade is already visible at $f=0.5$, for sufficiently

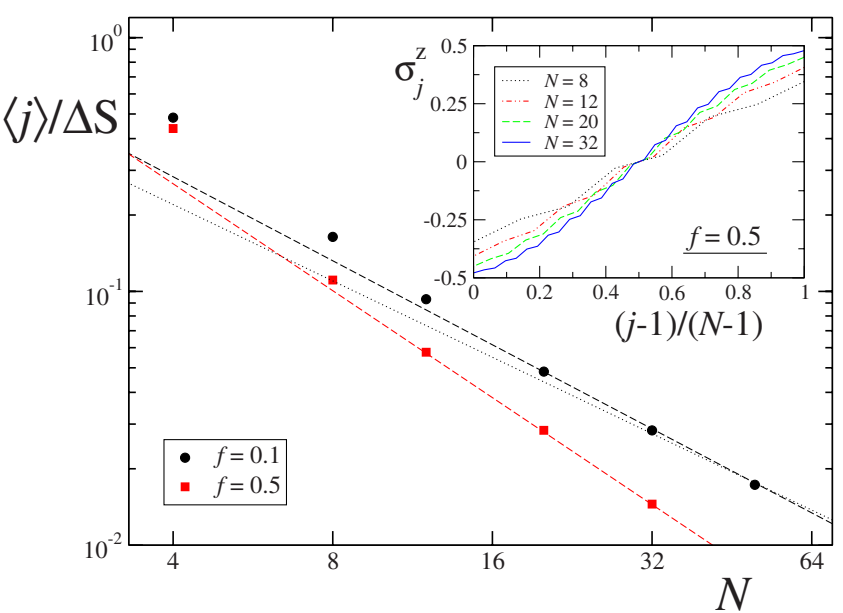

FIG. 15. (Color online) Dependence of the scaled spin current on the chain length in the staggered Heisenberg model, with $\Delta$ $=1.3, B=0.3$, and $\Gamma=1(\Delta S$ is the magnetization difference across the chain), at driving strength $f=0.1$ (circles) and $f=0.5$ (squares). The two dashed lines denote the behaviors $\sim 1.3 / N^{1.1}$ (for circles) and $\sim 1.85 / N^{1.4}$ (for squares), respectively, whereas dotted line indicates $1 / N$ behavior found in the linear response regime. In the inset we show magnetization profiles for $f=0.5$ and different system sizes. Data are obtained from MPO.

large sizes (see the inset of Fig. 15), similarly to what has been already discussed in Sec. V B 2.

On the other hand, we considered a situation in which the system does not exhibit NDC $(\Delta=0.5, B=0.5)$, but still it is quantum chaotic with respect to energy level statistics. In that case we found the usually predicted behavior for nonintegrable systems: ${ }^{20}$ the current is always proportional to the driving for any value of $f$ and a normal Ohmic regime for both small and strong drivings emerges, as shown in Fig. 16. This comes in sharp contrast to the integrable case, where at $\Delta<1$ ballistic spin transport takes place. Consistently with normal metallic conductors, the spin magnetization profiles exhibit a linear gradient (see the inset of Fig. 16).

From the results of this section we can conclude that the NDC phenomenon is governed by the anisotropy parameter $\Delta$ and is insensitive to the transition from integrability to quantum chaos. The appearance of NDC in the quantum chaos regime is particularly significant since in this case normal Ohmic conduction is expected in the linear response regime (small driving $f$ ). Due to the insulating behavior observed for $|\Delta|>1$ at large driving strengths $f$, the system should undergo a metal-to-insulator transition when increasing $f$.

\section{CONCLUSIONS}

In summary, we have performed extensive numerical simulations showing that the one-dimensional Hubbard model, as well as the corresponding spinless fermion model, exhibits a regime of negative differential conductivity, in which the particle current decreases as one increases the driving field. The same phenomenon, translated in terms of the spin current, is observed and studied also in the aniso- 


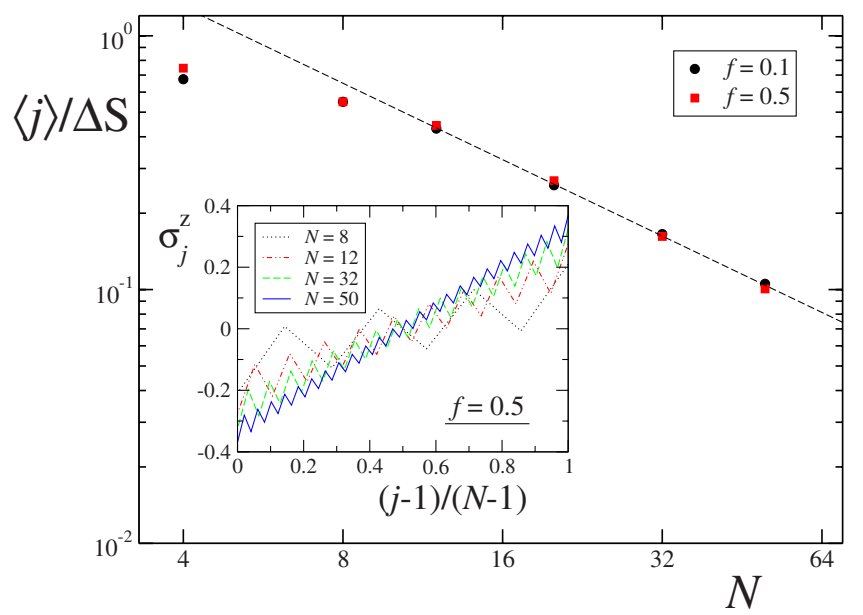

FIG. 16. (Color online) Dependence of the scaled spin current on the chain length for the staggered Heisenberg model, with $\Delta$ $=0.5, B=0.5$, and $\Gamma=1$. Both for strong and weak drivings the system shows a normal Ohmic behavior, as it is depicted by the straight dashed line, which corresponds to $\sim 5.2 / N$. The magnetization has an approximately linear profile, as shown in the inset, with a slight even-odd oscillation that is due to a staggered magnetic field. Data are obtained from MPO.

tropic Heisenberg spin chain. Our numerical data show that in the Ising-like regime, corresponding to anisotropy parameter $|\Delta|>1$, the system is a normal conductor for small driving fields, while it displays negative differential conductivity for stronger drivings. The phenomenon is stable against the breaking of integrability, as, for instance, it persists in the presence of a staggered magnetic field. On the other hand, in the $X Y$-like regime, $|\Delta|<1$, ballistic conduction with current proportional to driving field without negative differential conductivity is observed at all drivings. Negative differential conductivity is schematically explained using the spectrum of one-magnon excitations, which become localized for $|\Delta|>1$ at the chain boundaries.

The observed negative differential conductivity arises as an outcome of a beautiful interplay between coherent manybody quantum dynamics of the interacting electrons or spin chain and incoherent charge injection-extraction or spin pumping operated by macroscopic leads or spin baths. While our results are suggestive of a metal-insulator phase transition when driving our model systems far from equilibrium, new analytical approaches are required to ascertain whether NDC survives at the thermodynamic limit. At any rate, the discussed magnon-localization mechanism for NDC is of potential interest for nanoscale devices such as current or heat diodes and transistor.

Note added. After the completion of this work we became aware of a related paper ${ }^{60}$ where it is shown that negative differential conductivity can be also achieved in a continuum limit of a fermionic tight-binding model, driven far from equilibrium by embedding it between two free fermionic reservoirs.

\section{ACKNOWLEDGMENTS}

We thank G. Falci for useful discussions. T.P. and M.Ž. acknowledge support by Program P1-0044 of the Slovenian Research Agency.

\section{APPENDIX A: NUMERICAL METHODS}

\section{Quantum trajectory approach}

As compared to full density matrix simulations, the advantage of a quantum trajectory approach is that, instead of storing and evolving a density matrix of size $\mathcal{N} \times \mathcal{N}$, one works with a stochastically evolving state vector of size $\mathcal{N}$ $(\mathcal{N}$ being the Hilbert space dimension of the system). The first two terms in the right-hand side of Eq. (4) can be regarded as the evolution performed by an effective nonHermitian Hamiltonian $\mathcal{H}_{\text {eff }} \equiv \mathcal{H}_{S}+i \mathcal{K}$, with $\mathcal{K}=-\frac{1}{2} \Sigma_{m} L_{m}^{\dagger} L_{m}$; the last term is responsible for the so-called quantum jumps, as explained below. If the initial density matrix describes a pure state $\rho\left(t_{0}\right)=\left|\phi\left(t_{0}\right)\right\rangle\left\langle\phi\left(t_{0}\right)\right|$, after a small amount of time $d t$ it evolves into the statistical mixture

$$
\rho\left(t_{0}+d t\right)=\left(1-\sum_{m} d p_{m}\right)\left|\phi_{0}\right\rangle\left\langle\phi_{0}\left|+\sum_{m} d p_{m}\right| \phi_{m}\right\rangle\left\langle\phi_{m}\right|,
$$

where $d p_{m}=\left\langle\phi\left(t_{0}\right)\left|L_{m}^{\dagger} L_{m}\right| \phi\left(t_{0}\right)\right\rangle d t$ and the new states are defined by

$$
\left|\phi_{0}\right\rangle=\frac{e^{-i \mathcal{H}_{\mathrm{eff}} d t}\left|\phi\left(t_{0}\right)\right\rangle}{\sqrt{1-\sum_{m} d p_{m}}}, \quad\left|\phi_{m}\right\rangle=\frac{L_{m}\left|\phi\left(t_{0}\right)\right\rangle}{\| L_{m}\left|\phi\left(t_{0}\right)\right\rangle \|} .
$$

Therefore, with probability $d p_{m}$ a jump to the state $\left|\phi_{m}\right\rangle$ occurs, while with probability $1-\Sigma_{m} d p_{m}$ there are no jumps and the system evolves according to $\mathcal{H}_{\text {eff }}$.

In practice, one starts from a pure random state $\left|\phi\left(t_{0}\right)\right\rangle$ and, at intervals $d t$ much smaller than the relevant dynamical time scales, a random number $\varepsilon \in[0,1]$ is chosen. If $\varepsilon \leq \Sigma_{m} d p_{m}$, the state of the system jumps to one of the states $\left|\phi_{m}\right\rangle$ (to $\left|\phi_{1}\right\rangle$ if $0 \leq \varepsilon \leq d p_{1}$, to $\left|\phi_{2}\right\rangle$ if $d p_{1}<\varepsilon \leq d p_{1}+d p_{2}$, and so on). On the other hand, if $\varepsilon>\Sigma_{m} d p_{m}$ the evolution with the non-Hermitian Hamiltonian $\mathcal{H}_{\text {eff }}$ takes place, thus ending up in the state $\left|\phi_{0}\right\rangle$. This process has to be repeated as many times as $n_{\text {steps }}=T / d t$, where $T$ is the total evolution time. Assuming that there exists a single out-of-equilibrium steady state $\rho_{s}$, the expectation values $\langle A\rangle=\operatorname{tr}\left(A \rho_{s}\right)$ of any observable $A$ are obtained after averaging in time $\langle\phi(t)|A| \phi(t)\rangle$ up to a long enough time $T$, skipping the initial convergence time $T_{\text {conv }}$ needed for a solution of the Lindblad equation to converge into the stationary one.

In the cases discussed in this paper, the non-Hermitian evolution of $e^{-i \mathcal{H}_{\mathrm{eff}} d t}$ has been simulated with a second-order time Trotter expansion; a time slicing $d t \sim 2 \times 10^{-3}$ is sufficient to obtain accurate results. The fermionic systems taken into account are mapped into lattice spin models; simulations are then directly performed on spin-1/2 Hamiltonians [see Eqs. (14) and (18)]. We were able to simulate systems with sizes up to $\mathcal{N} \approx 10^{5}$ (that is, $N=\log _{2} \mathcal{N} \approx 16$ spin- $1 / 2$ particles). We calculated the stationary spin current $\langle j\rangle$ on the basis on Eq. (11). That is, we computed $\langle j\rangle$ by summing up all down-up flips minus all up-down flips at the right end of 
the chain or all up-down flips minus all down-up flips at the left end and then dividing by the simulation time. In the fermionic language, this corresponds to counting the number of electrons that enter the system minus the number of electrons that leave the system per unit time through the right electrode (emitter) or the number of electrons that leave the system minus the number of electrons that enter the system per unit time through the left electrode (collector). We also checked that the current obtained in this way is, up to statistical fluctuations due to finite integration times, equal to the one computed through Eqs. (15) and (19). A good convergence for $\langle j\rangle$ is already reached at $T_{\text {conv }} \sim 10^{4}$, while integration times $T$ of one order of magnitude longer are required in order to determine the stationary magnetization profiles (e.g., $T \sim 3 \times 10^{5}$ for $N=12$ in Fig. 6).

Data presented for the Hubbard model and the spinless fermion model with $N \leq 16$ have been obtained with this approach. For further details of the implementation of the quantum trajectory approach see Ref. 47.

\section{Matrix product operator formalism}

We have used the MPO ansatz for spin-1/2 particles. An arbitrary density matrix $\rho$ of a chain of $N$ spins $1 / 2$ can be expanded over all possible products of Pauli operators forming a basis of a $4^{N}$ dimensional Hilbert space of operators,

$$
|\rho\rangle=\sum_{\underline{s}} \alpha_{\underline{s}}\left|\sigma^{\underline{S}}\right\rangle,
$$

where we used the compact notation $\sigma^{\underline{s}}=\sigma_{1}^{s_{1}} \ldots \sigma_{N}^{s_{N}}, \underline{s}$ $\equiv s_{1} \ldots s_{N}$, and $s_{i} \in\{0,1,2,3\}$, with $\sigma^{0}=1, \sigma^{1}=\sigma^{x}, \sigma^{2}=\sigma^{y}$, $\sigma^{3}=\sigma^{z}$, and where lower indices in the Pauli operators denote the site number of the spin on which it operates. The use of ket notation in $|\rho\rangle$ outlines the fact that the density matrix $\rho$ can be seen as a vector in the operator Hilbert space spanned by the basis vectors $\left|\sigma^{s}\right\rangle$. In the MPO ansatz, the expansion coefficients $\alpha_{s}$ are expressed as traces of products of $N D \times D$ dimensional matrices $\mathbf{A}_{i}^{s_{i}}, i=1, \ldots, N$, as

$$
\alpha_{\underline{s}}=\operatorname{tr}\left(\mathbf{A}_{1}^{s_{1}} \ldots \mathbf{A}_{N}^{s_{N}}\right) .
$$

Thus, (density) operator (A3) is completely specified and thus parametrized in terms of a set of $4 N$ matrices $\mathbf{A}_{i}^{s_{i}}$, four for each site $i, s_{i}=0,1,2,3$. We note that these matrices are not related to physically observable quantities. The propagator corresponding to the master equation [Eq. (4)] is written as a product of propagators for small steps of length $d t$, typically $d t=10^{-1}$. Each small time-step propagator is then split using a third-order Trotter expansion into parts composed of mutually commuting two-spin terms. These nearestneighbor two-spin terms are then basic transformations performed within the MPO ansatz, namely, after each such twospin transformation, a singular value decomposition is performed in order to restore the shape of ansatz (A4). However, this step has to be combined with a truncation of the resulting matrices to a smaller fixed dimension $D$. Dimension $D$ is then chosen as a parameter by which we control the accuracy of the method. Note that the minimal necessary dimension $D$ is related to the bipartite entanglement of $|\rho\rangle$ in the Hilbert space of operators. This implies that MPO method will fail if the state $\rho$ builds up strong quantum correlations over large distances. For a review on MPO techniques see Ref. 61, while details of the implementation of the MPO method in quantum master equations can be found in Ref. 33.

Starting from an arbitrary initial density matrix $\rho\left(t_{0}\right)$ we are interested in the asymptotic nonequilibrium steady state $\rho_{s}$ reached after a sufficiently long time of simulation, i.e., of relaxation. Once $\rho_{s}$ is obtained, various expectation values can be evaluated. In particular, the spin current is obtained from Eq. (19) as $\langle j\rangle=J_{x} \operatorname{tr}\left[\rho_{s}\left(\sigma_{k}^{x} \sigma_{k+1}^{y}-\sigma_{k}^{y} \sigma_{k+1}^{x}\right)\right]$. The simulation time that is required to reach a stationary $\rho_{s}$ in the regime of NDC may greatly depend on the imposed gradient (driving field). In the most difficult situations that we studied here, the relaxation time after which the local current inhomogeneities decreased to a few percent was $T \sim 4000$. Note that this relaxation time is not directly comparable to the time needed in the quantum trajectory approach, as there large $T$ is needed also to perform statistical averaging. In the MPO approach averaging over Hilbert space is exact, up to the truncation imposed by finite matrix dimension $D$. The main advantage of MPO as compared to other approaches is that it generally enables simulation of larger systems. For small drivings one can go up to $N \sim 100$, while in the present work, where systems are driven far from equilibrium, we could reach sizes $N \sim 40$. Data shown for the spinless fermion model with $N>16$ have been obtained with MPO technique.

\section{APPENDIX B: MAPPING FERMIONIC SYSTEMS INTO SPIN CHAIN MODELS}

In this appendix we sketch the main steps of the JWT (Ref. 62) mapping the Hubbard and $t-V$ fermionic Hamiltonians with open boundary conditions into spin-1/2 ladder and chain models. In particular, we show that the bath operators injecting or extracting fermions at the two borders of the system correspond, in the spin-1/2 picture, to operators flipping the edge spins.

We start from the simpler case of the $t-V$ model, described by Hamiltonian (16). We first perform a JWT of fermions into hard-core bosons $\left(a_{j}^{\dagger}, a_{j}\right)$, defined by

$$
a_{j} \equiv \exp \left(i \pi \sum_{k<j} n_{k}\right) c_{j}=\left[\prod_{k=1}^{j-1}\left(1-2 n_{k}\right)\right] c_{j},
$$

where $n_{j}=c_{j}^{\dagger} c_{j}$ is the fermion number operator. The operators $a_{j}$ for different sites commute, but they are not ordinary bosonic operators since at most one boson is allowed on each site. One can indeed show that $\left(a_{j}^{\dagger}\right)^{2}|0\rangle=0$ and, on the same site, $\left\{a_{j}, a_{j}^{\dagger}\right\}=1$. Moreover, by using

$$
\prod_{k=1}^{j-1}\left(1-2 n_{k}\right) \prod_{k^{\prime}=1}^{j}\left(1-2 n_{k^{\prime}}\right)=1-2 n_{j}
$$

[it follows from $\left.\left(1-2 n_{k}\right)\left(1-2 n_{k}\right)=1\right]$ and the fact that terms with different site indices commute, we find $n_{j} \equiv c_{j}^{\dagger} c_{j}=a_{j}^{\dagger} a_{j}$ and $c_{j}^{\dagger} c_{j+1}=c_{j}^{\dagger}\left(1-2 n_{j}\right) c_{j+1}=a_{j}^{\dagger} a_{j+1}$.

Then we have to transform the hard-core bosons into spin-1/2 particles in a representation that identifies, at 
each site, the state $|0\rangle \equiv a|1\rangle$ with $|\downarrow\rangle$ and $|1\rangle \equiv a^{\dagger}|0\rangle$ with $|\uparrow\rangle$. If $\sigma_{j}^{\alpha}$ denote the corresponding spin- $1 / 2$ particles (which, of course, obey the standard anticommutation rules $\left.\left\{\sigma_{j}^{-}, \sigma_{j^{\prime}}^{+}\right\}=\delta_{j, j^{\prime}}\right)$, the explicit mapping is given by

$$
\begin{gathered}
a_{j}^{\dagger}=\sigma_{j}^{+}, \\
a_{j}=\sigma_{j}^{-}, \\
2 a_{j}^{\dagger} a_{j}=\sigma_{j}^{z}+1 .
\end{gathered}
$$

This leads quite straightforwardly to the following expressions:

$$
\begin{gathered}
n_{j} n_{j+1}=a_{j}^{\dagger} a_{j} a_{j+1}^{\dagger} a_{j+1}=\frac{1}{4}\left(\sigma_{j}^{z} \sigma_{j+1}^{z}+\sigma_{j}^{z}+\sigma_{j+1}^{z}+1\right), \\
c_{j}^{\dagger} c_{j+1}+c_{j+1}^{\dagger} c_{j}=a_{j}^{\dagger} a_{j+1}+a_{j+1}^{\dagger} a_{j}=\frac{1}{2}\left(\sigma_{j}^{x} \sigma_{j+1}^{x}+\sigma_{j}^{y} \sigma_{j+1}^{y}\right) .
\end{gathered}
$$

Substituting them in Eq. (16) with open boundary conditions we finally get

$$
\begin{aligned}
\mathcal{H}_{S}= & -\frac{t}{2} \sum_{j=1}^{N-1}\left(\sigma_{j}^{x} \sigma_{j+1}^{x}+\sigma_{j}^{y} \sigma_{j+1}^{y}\right)+\frac{V}{4} \sum_{j=1}^{N-1} \sigma_{j}^{z} \sigma_{j+1}^{z} \\
& -\frac{V}{4}\left[\sigma_{1}^{z}+\sigma_{N}^{z}-(N-1)-2 \sum_{j=1}^{N} \sigma_{j}^{z}\right],
\end{aligned}
$$

which is the Heisenberg Hamiltonian with $J_{x} \equiv-t / 2$ and $J_{z} \equiv V / 4$ plus an on-site transverse uniform magnetic field of strength $V / 2$ and local transverse fields at the edge spins of the chain.

The Lindblad operators are mapped into spin operators that flip the outer spins. Indeed we have $c_{1}^{\dagger}=\sigma_{1}^{+}$and $c_{1}=\sigma_{1}^{-}$ on the left side and

$$
c_{N}^{\dagger}=e^{i \pi \mathcal{N}_{F}^{(1)}} \sigma_{N}^{+}
$$

and $c_{N}=e^{i \pi \mathcal{N}_{F}^{(1)}} \sigma_{N}^{-}$on the right side (where $\mathcal{N}_{F}^{(k)}$ is the number of fermions in the leftmost $N-k$ sites of the chain). Since after action of the operator $\sigma_{N}^{+}$in Eq. (B6) the rightmost site is always occupied, we can just as well rewrite Eq. (B6) as

$$
c_{N}^{\dagger}=-e^{i \pi \mathcal{N}_{F}^{(0)}} \sigma_{N}^{+},
$$

where $\mathcal{N}_{F}^{(0)}$ is the total number of spin up (occupied fermion states) in the entire lattice.

Now, the crucial observation is that $e^{i \pi \mathcal{N}_{F}^{(0)}}$ is an operator that commutes (anticommutes) with the fermionic algebra consisting even (odd) number of fermionic operators. Since all the terms in our Lindblad equations conserve the parity of density operators, ${ }^{26}$ i.e., they map, in the Liouville space sense, the products of even or odd number of fermionic operators $c_{j}, c_{j}^{\dagger}$ into products of even or odd number of such operators, the Lindblad master equation (4) can be restricted to even-parity density operator subspace only. Thus, the factor $e^{i \pi \mathcal{N}_{F}^{(0)}}$ cancels from the Lindblad equation even though the number of particles (or magnetization in the spin lan-

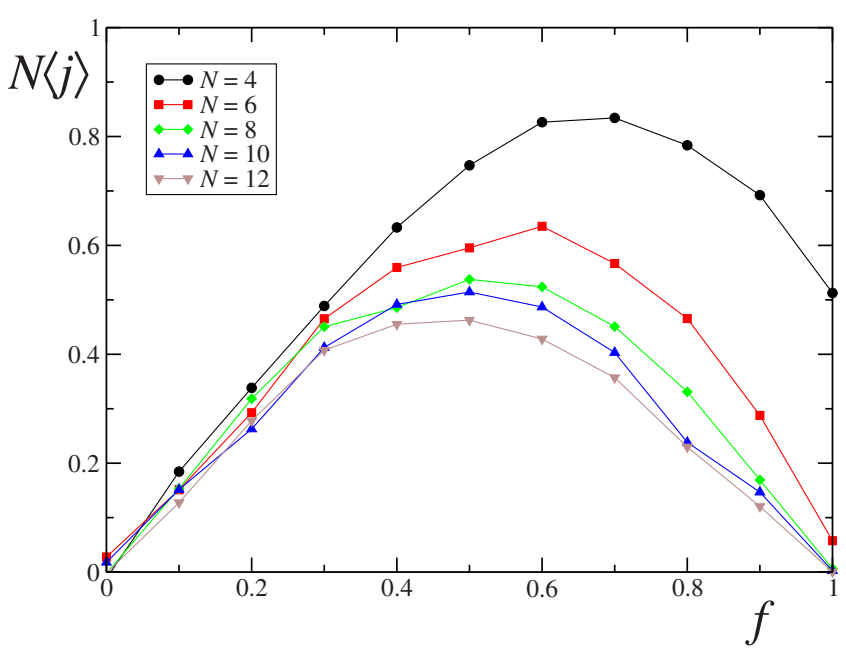

FIG. 17. (Color online) Spin current as a function of the driving strength for the Hamiltonian in Eq. (B5) with $t=2, V=8$ (that corresponds to $J_{x}=1, J_{z}=2$, so that $\Delta=2$ ). In contrast to the standard XXZ model, here we added a uniform transverse magnetic field of intensity $V / 2$ plus two transverse fields of strength $-V / 4$ at the border sites of the Heisenberg chain. The system-bath coupling is set equal to $\Gamma=4$; the simulation time (QT approach) is $T=2.5$ $\times 10^{4}$.

guage) is not conserved in an open system. Odd-parity density operator subspace, which would include terms like $\mid$ odd $\rangle\langle$ even|, etc., which change the number of fermions by an even number, could be studied with a similar (but not identical) Lindblad equation with an additional minus sign in all the Lindblad terms. However, since all the physical observables in question, say $A=j_{k}, n_{k}, \ldots$, are represented as products of even number of fermionic operators, only the even-parity component of the density operator affects the expectation values $\langle A\rangle=\operatorname{tr}[\rho A]$. Thus we can safely forget the phase operator $e^{i \pi \mathcal{N}_{F}^{(0)}}$ and map Lindblad operators from fermionic language to spin language in the Lindblad master equation [Eq. (4)] as $c_{j}^{\dagger} \rightarrow \sigma_{j}^{+}$and $c_{j} \rightarrow \sigma_{j}^{-}$, keeping in mind the issue of operator-space parity in case expectation values of odd-parity operators would be needed.

The charge current becomes then a spin current, while $\frac{1}{2}\left(1+\left\langle\sigma_{j}^{z}\right\rangle\right)$ is the charge density on site $j$. We numerically checked that the addition of two local transverse fields on the border sites and of a uniform magnetic field does not qualitatively affect the NDC effect, as shown in Fig. 17.

We now show that the Hubbard model of Eq. (12) can be mapped into the Heisenberg spin ladder of Eq. (14). Following the same steps for the $t$ - $V$ model, one first performs a double JWT of spin-up and spin-down fermions into two species of hard-core bosons $\left(a_{j}^{\dagger}, a_{j}\right)$ and $\left(b_{j}^{\dagger}, b_{j}\right)$. Such transformation is defined by

$$
\begin{gathered}
a_{j} \equiv \exp \left(i \pi \sum_{k<j} n_{k, \uparrow}\right) c_{j, \uparrow}, \\
b_{j} \equiv \exp \left(i \pi \sum_{k<j} n_{k, \downarrow}\right) c_{j, \downarrow} .
\end{gathered}
$$

Then the hard-core bosons are transformed into two species of spin-1/2 particles $\left(\sigma_{j}\right.$ and $\left.\tau_{j}\right)$, in a representation analo- 

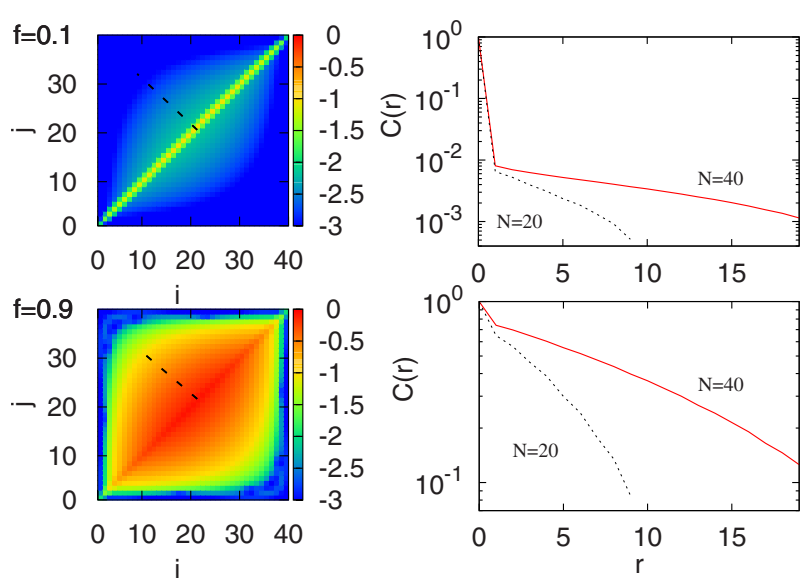

FIG. 18. (Color online) Spin-spin correlation function $C(i, j)$ in a chain with 40 spins for $f=0.1$ (top row) and $f=0.9$ (bottom row); here we set $\Delta=2$ and $\Gamma=4$. The code in the left panels denotes $\log _{10}[C(i, j)]$. The right plots display correlation function $C(r \equiv \mid i$ $-j \mid$ ) along the diagonal denoted by a dashed line in the left plots; the case with $N=20$ spins is also shown. Data are obtained using the MPO ansatz.

gous to that of Eqs. (B3). Proceeding along the same transformations as before and using $n_{j, \uparrow} n_{j, \downarrow}=\frac{1}{2}\left(\sigma_{j}^{z}+1\right) \frac{1}{2}\left(\tau_{j}^{z}+1\right)$, we finally arrive at spin ladder Hamiltonian (14). In complete analogy with the $t-V$ model, the Lindblad operators of Eqs. (5) and (6) are mapped into spin operators flipping the outer spins of the ladder.

\section{APPENDIX C: SPIN-SPIN CORRELATIONS}

We provide here some numerical data on the behavior of the steady-state spin-spin correlation functions for the Heisenberg chain driven far from equilibrium, aimed at investigating the emergence, in the gapped regime and at strong driving strength $f$, of a long-range correlation order. ${ }^{22}$ The steady-state spin-spin correlation function is defined as

$$
C(i, j)=\left\langle\sigma_{i}^{z} \sigma_{j}^{z}\right\rangle-\left\langle\sigma_{i}^{z}\right\rangle\left\langle\sigma_{j}^{z}\right\rangle,
$$

where $\left\langle\sigma_{i}^{z} \sigma_{j}^{z}\right\rangle=\operatorname{tr}\left(\sigma_{i}^{z} \sigma_{j}^{z} \rho_{s}\right)$ and $\left\langle\sigma_{i}^{z}\right\rangle=\operatorname{tr}\left(\sigma_{i}^{z} \rho_{s}\right)$, while averages are taken on the steady state. Results are plotted in Fig. 18. With increasing $f$ and above $f^{*}$, a dramatic slowing down of the correlation decay is apparent. Unfortunately, a more quantitative understanding of a possible critical behavior in the large- $f$ regime is at present out of our capabilities since it would require the analysis of much bigger systems, and this is numerically not accessible for the model under consideration.

\section{APPENDIX D: SOLUTION OF THE ONE-MAGNON MODEL}

The matrix associated with Hamiltonian (18) in the onemagnon basis $\{|1\rangle,|2\rangle, \ldots,|N\rangle\}$ is tridiagonal and reads as follows:

$$
H=\left[\begin{array}{ccccccccc}
\alpha^{\prime} & \beta & 0 & & & \ldots & & & 0 \\
\beta & \alpha & \beta & 0 & & \ldots & & & 0 \\
0 & \beta & \alpha & \beta & 0 & \ldots & & & 0 \\
\vdots & & & & & & & \vdots \\
0 & & & & & \ldots & \beta & \alpha & \beta \\
0 & & & & & \ldots & 0 & \beta & \alpha^{\prime}
\end{array}\right],
$$

where $\alpha^{\prime} \equiv(N-3) J_{z}, \alpha \equiv(N-5) J_{z}$, and $\beta \equiv 2 J_{x}$. Note that the overall magnetization is conserved by the XXZ Hamiltonian, so the states corresponding to the other spin sectors are not coupled (by the autonomous evolution) to the one-magnon sector. The eigenvalues of $H$ are given by the roots of the characteristic polynomial $D_{N}(E) \equiv \operatorname{det}(E \rrbracket-H)$.

First, it is convenient to solve the eigenvalue problem for $\alpha=\alpha^{\prime}$. We define $\left.D_{N}^{(0)} \equiv D_{N}\right|_{\alpha=\alpha^{\prime}}$. The following recurrence relation holds:

$$
D_{j}^{(0)}=(E-\alpha) D_{j-1}^{(0)}-\beta^{2} D_{j-2}^{(0)} .
$$

The general solution to this difference equation is

$$
D_{m}^{(0)}=\beta^{m}\left(A e^{i m \phi}+B e^{-i m \phi}\right),
$$

with

$$
\cos \phi \equiv\left(\frac{E-\alpha}{2 \beta}\right)
$$

and the constants $A$ and $B$ determined from the conditions

$$
\begin{gathered}
D_{1}^{(0)}=E-\alpha, \\
D_{2}^{(0)}=(E-\alpha) D_{1}^{(0)}-\beta^{2} .
\end{gathered}
$$

We finally obtain

$$
D_{N}^{(0)}=\frac{\beta^{N} \sin [(N+1) \phi]}{\sin \phi},
$$

whose solutions are

$$
E_{m}^{(0)}=\alpha+2 \beta \cos \left(\frac{m \pi}{N+1}\right), \quad m=1, \ldots, N .
$$

These eigenvalues are located in the energy band $\alpha-2|\beta|$ $<E<\alpha+2|\beta|$. Therefore, $\phi$ is always real and the eigenstates ("Bloch orbitals")

$$
\left|\psi_{m}^{(0)}\right\rangle=\sqrt{\frac{2}{N+1}}_{k=1}^{N} \sin \left(\frac{\pi m k}{N+1}\right)|k\rangle,
$$

corresponding to the eigenvalues $E_{m}^{(0)}$ are delocalized along the spin chain.

In the case $\alpha \neq \alpha^{\prime}$ we obtain

$$
\begin{aligned}
D_{N} & =D_{N}^{(0)}+2\left(\alpha-\alpha^{\prime}\right) D_{N-1}^{(0)}+\left(\alpha-\alpha^{\prime}\right)^{2} D_{N-2}^{(0)} \\
& =\frac{\beta^{N}}{\sin \phi}\left\{\sin [(N+1) \phi]-2 \Delta \sin (N \phi)+\Delta^{2} \sin [(N-1) \phi]\right\},
\end{aligned}
$$

where we have used 


$$
\frac{\alpha^{\prime}-\alpha}{\beta}=\frac{J_{z}}{J_{x}}=\Delta .
$$

The equation $D_{N}=0$ can be analytically solved for large $N$. There always exist at least $N-2$ delocalized solutions lying in the energy band between $\alpha-2|\beta|$ and $\alpha+2|\beta|$. The "molecular orbitals" $\left|\psi_{ \pm}\right\rangle \approx \frac{1}{\sqrt{2}}\left(\left|\psi_{L}\right\rangle \pm\left|\psi_{R}\right\rangle\right)$ appear when $|\Delta|>1$.

If $\Delta>1$, we have $\phi=i \chi(\chi \in R), e^{\chi} \approx \Delta$,

$$
\begin{aligned}
\left|\psi_{L}\right\rangle & \approx \sqrt{\frac{1-e^{-2 \chi}}{1-e^{-2 N \chi}} \sum_{m=1}^{N} e^{-(m-1) \chi}|m\rangle,} \\
\left|\psi_{R}\right\rangle & \approx \sqrt{\frac{1-e^{-2 \chi}}{1-e^{-2 N \chi}}} \sum_{m=1}^{N} e^{-(N-m) \chi}|m\rangle .
\end{aligned}
$$

The states $\left|\psi_{L, R}\right\rangle$ are centered at sites 1 and $N$, respectively, and their localization length $\ell \approx 1 / \chi=1 / \ln (\Delta)$. The corresponding eigenvalues are given by

$$
E_{1} \approx E_{N} \approx \alpha+\beta\left(\Delta+\frac{1}{\Delta}\right) .
$$

If $\Delta<-1, \phi=i \chi+\pi(\chi \in \mathbb{R}), e^{\chi} \approx-\Delta$,

$$
\begin{aligned}
\left|\psi_{L}\right\rangle & \approx \sqrt{\frac{1-e^{-2 \chi}}{1-e^{-2 N \chi}}} \sum_{m=1}^{N}(-1)^{m} e^{-(m-1) \chi}|m\rangle, \\
\left|\psi_{R}\right\rangle & \approx \sqrt{\frac{1-e^{-2 \chi}}{1-e^{-2 N \chi}}} \sum_{m=1}^{N}(-1)^{m} e^{-(N-m) \chi}|m\rangle .
\end{aligned}
$$

These states have localization length $\ell \approx 1 / \chi=1 / \ln (-\Delta)$. The corresponding eigenvalues are again given by Eq. (D12).

The gap $\delta_{E}$ between the energy levels $E_{1}$ and $E_{N}$ shrinks exponentially with the system size:
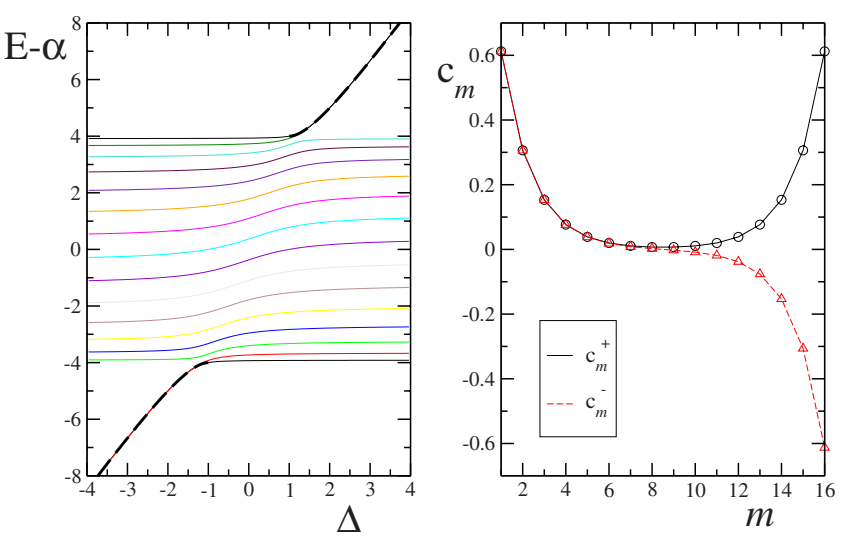

FIG. 19. (Color online) The one-magnon model for $N=16$ spins. Left: energy spectrum as a function of $\Delta$; large- $N$ analytic result (D12) is shown by thick dashed lines. Right: molecular states $\left|\psi_{ \pm}\right\rangle=\Sigma_{m=1}^{N}\left(c_{m}\right)_{ \pm}|m\rangle$ at $\Delta=2$; the numerically computed coefficients $\left(c_{m}\right)_{+}$and $\left(c_{m}\right)_{-}$are shown as circles and triangles; the large- $N$ analytic results are shown as full and dashed curves.

$$
\delta_{E} \propto\left|\left\langle\psi_{L} \mid \psi_{R}\right\rangle\right| \approx \exp \left(-\frac{N}{\ell}\right),
$$

so that the coherent tunneling between sites 1 and $N$ requires a time scale that grows exponentially with $N$. Therefore, for the purposes of our present investigation we can say that a spin-flip excitation created at one boundary of a ferromagnetic domain remains in practice exponentially localized over a length $\ell=1 / \ln |\Delta|$.

Numerical illustrations, for a chain of $N=16$ spins, of the appearance, for $\Delta \mid>1$, of two states outside the energy band $(\alpha-2|\beta|, \alpha+2|\beta|)$ and of the molecular orbitals $\left|\psi_{ \pm}\right\rangle$, are provided in Fig. 19.

Finally, we note that the one-magnon-localization model discussed in this section has deep similarities with a tightbinding model discussed in the context of surface physics. ${ }^{63}$
${ }^{1}$ Strong Interactions in Low Dimensions, edited by D. Baeriswyl and L. Degiorgi (Kluwer Academic, Dordrecht, 2004).

${ }^{2}$ C. Joachim, J. K. Gimzewski, and A. Aviram, Nature (London) 408, 541 (2000).

${ }^{3}$ A. Nitzan and M. A. Ratner, Science 300, 1384 (2003).

${ }^{4}$ Introducing Molecular Electronics, edited by G. Cuniberti, G. Fagas, and K. Richter (Springer-Verlag, Berlin, 2005).

${ }^{5}$ J. Park, A. N. Pasupathy, J. I. Goldsmith, C. Chang, Y. Yaish, J. R. Petta, M. Rinkoski, J. P. Sethna, H. D. Abruña, P. L. McEuen, and D. C. Ralph, Nature (London) 417, 722 (2002).

${ }^{6}$ J. Nygård, D. H. Cobden, and P. E. Lindelof, Nature (London) 408, 342 (2000).

${ }^{7}$ J. Weis, R. J. Haug, K. v. Klitzing, and K. Ploog, Phys. Rev. Lett. 71, 4019 (1993).

${ }^{8}$ C. Zhou, J. Kong, E. Yenilmez, and H. Dai, Science 290, 1552 (2000).

${ }^{9}$ J. Chen, M. A. Reed, A. M. Rawlett, and J. M. Tour, Science 286, 1550 (1999).
${ }^{10}$ D. V. Averin, A. N. Korotkov, and K. K. Likharev, Phys. Rev. B 44, 6199 (1991).

${ }^{11}$ C. W. J. Beenakker, Phys. Rev. B 44, 1646 (1991).

${ }^{12}$ D. Weinmann, W. Häusler, and B. Kramer, Phys. Rev. Lett. 74, 984 (1995).

${ }^{13}$ A. Thielmann, M. H. Hettler, J. König, and G. Schön, Phys. Rev. B 71, 045341 (2005).

${ }^{14}$ F. Elste and C. Timm, Phys. Rev. B 73, 235305 (2006).

${ }^{15}$ F. Meier and D. Loss, Phys. Rev. Lett. 90, 167204 (2003).

${ }^{16}$ X. Zotos, Phys. Rev. Lett. 82, 1764 (1999).

${ }^{17}$ P. Prelovšek, S. El Shawish, X. Zotos, and M. Long, Phys. Rev. B 70, 205129 (2004).

${ }^{18}$ F. Heidrich-Meisner, A. Honecker, D. C. Cabra, and W. Brenig, Phys. Rev. B 68, 134436 (2003).

${ }^{19}$ B. N. Narozhny, A. J. Millis, and N. Andrei, Phys. Rev. B 58, R2921 (1998).

${ }^{20}$ H. Castella, X. Zotos, and P. Prelovšek, Phys. Rev. Lett. 74, 972 (1995). 
${ }^{21}$ X. Zotos and P. Prelovšek, Phys. Rev. B 53, 983 (1996).

${ }^{22}$ T. Prosen and I. Pižorn, Phys. Rev. Lett. 101, 105701 (2008).

${ }^{23}$ M. J. Henrich, G. Mahler, and M. Michel, Phys. Rev. E 75, 051118 (2007).

${ }^{24}$ Y. Yan, C.-Q. Wu, and B. Li, Phys. Rev. B 79, 014207 (2009).

${ }^{25}$ D. Burgarth and V. Giovannetti, Phys. Rev. Lett. 99, 100501 (2007); in Quantum Information and Many Body Quantum Systems, edited by M. Ericsson and S. Montangero (Edizioni della Normale, Pisa, 2008), p. 17.

${ }^{26}$ T. Prosen, New J. Phys. 10, 043026 (2008).

${ }^{27}$ K. Saito, S. Takesue, and S. Miyashita, Phys. Rev. E 61, 2397 (2000).

${ }^{28}$ K. Saito, Europhys. Lett. 61, 34 (2003).

${ }^{29}$ M. Michel, M. Hartmann, J. Gemmer, and G. Mahler, Eur. Phys. J. B 34, 325 (2003).

${ }^{30}$ H. Wichterich, M. J. Henrich, H.-P. Breuer, J. Gemmer, and M. Michel, Phys. Rev. E 76, 031115 (2007); M. Michel, O. Hess, H. Wichterich, and J. Gemmer, Phys. Rev. B 77, 104303 (2008).

${ }^{31}$ C. Mejía-Monasterio, T. Prosen, and G. Casati, EPL 72, 520 (2005).

${ }^{32}$ G. Benenti, G. Casati, T. Prosen, and D. Rossini, EPL 85, 37001 (2009).

${ }^{33}$ T. Prosen and M. Žnidarič, J. Stat. Mech.: Theory Exp. (2009) P02035.

${ }^{34}$ L. Mühlbacher and E. Rabani, Phys. Rev. Lett. 100, 176403 (2008).

${ }^{35}$ S. Langer, F. Heidrich-Meisner, J. Gemmer, I. P. McCulloch, and U. Schollwoeck, Phys. Rev. B 79, 214409 (2009).

${ }^{36}$ W. Li, G. Xianlong, C. Kollath, and M. Polini, Phys. Rev. B 78, 195109 (2008).

${ }^{37}$ G. Xianlong, M. Polini, D. Rainis, M. P. Tosi, and G. Vignale, Phys. Rev. Lett. 101, 206402 (2008).

${ }^{38}$ L. Bogani and W. Wernsdorfer, Nature Mater. 7, 179 (2008).

${ }^{39}$ A. Ghirri, A. Candini, M. Evangelisti, M. Affronte, S. Carretta, P. Santini, G. Amoretti, R. S. G. Davies, G. Timco, and R. E. P. Winpenny, Phys. Rev. B 76, 214405 (2007).

${ }^{40}$ H.-P. Breuer and F. Petruccione, The Theory of Open Quantum Systems (Oxford University Press, Oxford, 2002).

${ }^{41}$ S. A. Gurvitz and Ya. S. Prager, Phys. Rev. B 53, 15932 (1996).
${ }^{42}$ X.-Q. Li, J. Y. Luo, Y.-G. Yang, P. Cui, and Y. J. Yan, Phys. Rev. B 71, 205304 (2005).

${ }^{43}$ U. Harbola, M. Esposito, and S. Mukamel, Phys. Rev. B 74, 235309 (2006).

${ }^{44}$ J. Dalibard, Y. Castin, and K. Mølmer, Phys. Rev. Lett. 68, 580 (1992).

${ }^{45}$ M. B. Plenio and P. L. Knight, Rev. Mod. Phys. 70, 101 (1998).

${ }^{46}$ T. A. Brun, Am. J. Phys. 70, 719 (2002).

${ }^{47}$ G. G. Carlo, G. Benenti, and G. Casati, Phys. Rev. Lett. 91, 257903 (2003); G. G. Carlo, G. Benenti, G. Casati, and C. Mejía-Monasterio, Phys. Rev. A 69, 062317 (2004).

${ }^{48}$ G. Vidal, Phys. Rev. Lett. 91, 147902 (2003); 93, 040502 (2004).

${ }^{49}$ S. R. White and A. E. Feiguin, Phys. Rev. Lett. 93, 076401 (2004).

${ }^{50}$ F. Verstraete, J. J. Garcia-Ripoll, and J. I. Cirac, Phys. Rev. Lett. 93, 207204 (2004).

${ }^{51}$ M. Zwolak and G. Vidal, Phys. Rev. Lett. 93, 207205 (2004).

${ }^{52}$ A. J. Daley, C. Kollath, U. Schollwöck, and G. Vidal, J. Stat. Mech.: Theory Exp. (2004) P04005.

${ }^{53}$ S. Lepri, R. Livi, and A. Politi, Phys. Rep. 377, 1 (2003).

${ }^{54}$ N. Motoyama, H. Eisaki, and S. Uchida, Phys. Rev. Lett. 76, 3212 (1996).

${ }^{55}$ X. Zotos, F. Naef, and P. Prelovšek, Phys. Rev. B 55, 11029 (1997).

${ }^{56}$ K. Saito, S. Takesue, and S. Miyashita, Phys. Rev. E 54, 2404 (1996).

${ }^{57}$ P. Mazur, Physica (Amsterdam) 43, 533 (1969).

${ }^{58}$ F. Haake, Quantum Signatures of Chaos, 2nd ed. (Springer, Berlin, 2001)

${ }^{59}$ T. Guhr, A. Müller-Groeling, and H. A. Weidenmüller, Phys. Rep. 299, 189 (1998)

${ }^{60}$ S. Ajisaka, H. Nishimura, S. Tasaki, and I. Terasaki, Prog. Theor. Phys. 121, 1289 (2009).

${ }^{61}$ F. Verstraete, J. I. Cirac, and V. Murg, Adv. Phys. 57, 143 (2008).

${ }^{62}$ E. Lieb, T. Schultz, and D. Mattis, Ann. Phys. 16, 407 (1961).

${ }^{63}$ T. B. Grimley, Adv. Catal. 12, 1 (1960); G. P. Brivio and T. B. Grimley, J. Phys. C 10, 2351 (1977). 